\title{
THE REVERSE ORDER LAWS AND THE MIXED-TYPE REVERSE ORDER LAWS FOR GENERALIZED INVERSES OF MULTIPLE MATRIX PRODUCTS*
}

\author{
ZHIPING XIONG ${ }^{\dagger}$ AND BING ZHENG Z $^{\ddagger}$
}

\begin{abstract}
In this paper, necessary and sufficient conditions for a number of reverse order laws and mixed-type reverse order laws are derived by using the maximal ranks of the generalized Schur complements.
\end{abstract}

Key words. Reverse order law, Mixed-type reverse order law, Generalized inverse, Matrix product, Maximal rank, Generalized Schur complement.

AMS subject classifications. 15A03, 15A09.

1. Introduction. Let $\mathbb{C}^{m \times n}$ denote the set of $m \times n$ matrices with complex entries and $\mathbb{C}^{m}$ denote the set of $m$-dimensional vectors. $I_{k}$ denotes the identity matrix of order $k, O_{m \times n}$ is the $m \times n$ matrix of all zero entries (if no confusion occurs, we will drop the subindex). For a matrix $A \in \mathbb{C}^{m \times n}, A^{*}, R(A)$ and $r(A)$ denote the conjugate transpose, the range space and the rank of the matrix $A$, respectively.

We recall that a generalized inverse $X \in \mathbb{C}^{n \times m}$ of a given matrix $A \in \mathbb{C}^{m \times n}$ is a matrix which satisfies some of the following four Penrose equations [2]:
(1) $A X A=A$,
(2) $X A X=X$,
(3) $(A X)^{*}=A X$,
(4) $(X A)^{*}=X A$.

For any matrix $A \in \mathbb{C}^{m \times n}$, let $A\{i, j, \ldots, k\}$ denote the set of matrices $X \in \mathbb{C}^{n \times m}$ which satisfy equations $(i),(j), \ldots,(k)$ from among equations $(1)--(4)$ of (1.1). A matrix in $A\{i, j, \ldots, k\}$ is called an $\{i, j, \ldots, k\}$-inverse of $A$ and denoted by $A^{(i, j, \ldots, k)}$. In particular, an $n \times m$ matrix $X$ of the set $A\{1\}$ is called a $\{1\}$-inverse or a $g$-inverse of $A$. $X$ is called a $\{1,3\}$-inverse or a least squares $g$-inverse of $A$ if it is an element

\footnotetext{
${ }^{*}$ Received by the editors on January 18, 2011. Accepted for publication on July 2, 2011. Handling Editor: Oskar Maria Baksalary.

${ }^{\dagger}$ School of Mathematics and Statistics, Lanzhou University, Lanzhou 730000, P.R. China (Present address: Department of Mathematics and Physics, Wuyi University, Jiangmen 529020, P.R. China (xzpwhere@163.com)).

${ }^{\ddagger}$ School of Mathematics and Statistics, Lanzhou University, Lanzhou 730000, P.R. China (bzheng@lzu.edu.cn). Supported by the Fundamental Research Fund for Physics and Mathematic of Lanzhou University, the Gansu Project of Science and Technology (no. 096RJZA049), the National Natural Science Foundation of China (no. 11171371), and the Fundamental Research Funds for the Central Universities (no. lzujbky-2009-49), China.
} 
of $A\{1,3\} ; X$ is called a $\{1,4\}$-inverse or a minimum norm $g$-inverse of $A$ if it is an element of $A\{1,4\}$. The Moore-Penrose inverse of $A$ is an element of the set $A\{1,2,3,4\}$. Any matrix $A$ admits a unique Moore-Penrose inverse, denoted by $A^{\dagger}$. We refer the readers to $[2,15]$ for basic results on the generalized inverses.

Let $A_{i}, i=1,2, \ldots, n$, be $n$ matrices such that the product $A_{1} A_{2} \cdots A_{n}$ exists. If each of the $n$ matrices is nonsingular, then the product $A_{1} A_{2} \cdots A_{n}$ is nonsingular too, and the inverse of $A_{1} A_{2} \cdots A_{n}$ satisfies the reverse order law $\left(A_{1} A_{2} \cdots A_{n}\right)^{-1}=$ $A_{n}^{-1} A_{n-1}^{-1} \cdots A_{1}^{-1}$. However, this reverse order law does not hold for generalized inverses. Hence the necessary and sufficient conditions for the reverse order laws of the generalized inverses of multiple matrix products to hold yield a class of interesting problems that are fundamental in the theory of generalized inverses of matrices and statistics. They have attracted considerable attention since the middle $1960 \mathrm{~s}$, and recently many interesting results have been obtained, see $[1,3,4,5,6,8,9,10,13$, $14,16,17,18,19,20,21]$.

In the paper [6], Liu and Wei studied the reverse order law for least squares $g$-inverses of multiple matrix products and derived some necessary and sufficient conditions for

$$
A_{n}\{1,3\} A_{n-1}\{1,3\} \cdots A_{1}\{1,3\} \subseteq\left(A_{1} A_{2} \cdots A_{n}\right)\{1,3\}
$$

and

$$
A_{n}\{1,4\} A_{n-1}\{1,4\} \cdots A_{1}\{1,4\} \subseteq\left(A_{1} A_{2} \cdots A_{n}\right)\{1,4\}
$$

by using P-SVD (Product Singular Value Decomposition). In this paper we revisit these two reverse order laws by using the maximal rank of the generalized Schur complements [12]. Some new simpler equivalent conditions for the inclusions (1.2) and (1.3) are obtained in terms of only the ranks of the known matrices. Compared with the conditions given in [6], our conditions can be easily checked and their proofs are very simple. Furthermore, with the same technique the necessary and sufficient conditions for the following mixed-type reverse order laws are derived:

$$
\begin{gathered}
A_{n}\{1,3\} A_{n-1}\{1,3\} \cdots A_{1}\{1,3\} \subseteq\left(A_{1} A_{2} \cdots A_{n}\right)\{1\}, \\
A_{n}\{1,4\} A_{n-1}\{1,4\} \cdots A_{1}\{1,4\} \subseteq\left(A_{1} A_{2} \cdots A_{n}\right)\{1\}, \\
A_{n}\{1\} A_{n-1}\{1\} \cdots A_{1}\{1\} \subseteq\left(A_{1} A_{2} \cdots A_{n}\right)\{1,3\},
\end{gathered}
$$

and

$$
A_{n}\{1\} A_{n-1}\{1\} \cdots A_{1}\{1\} \subseteq\left(A_{1} A_{2} \cdots A_{n}\right)\{1,4\} .
$$


For the sake of the simplicity in the following discussion, we will adopt the following notations for the matrix products with $A_{i} \in \mathbb{C}^{l_{i} \times l_{i+1}}$ and $X_{i} \in \mathbb{C}^{l_{i+1} \times l_{i}}$, $i=1,2, \ldots, n$ :

$$
\mathscr{A}_{i}^{j}=A_{i} A_{i+1} \cdots A_{j}, \quad \mathscr{X}_{i}^{j}=X_{i}^{*} X_{i+1}^{*} \cdots X_{j}^{*}, \quad 1 \leq i \leq j \leq n .
$$

In particular, $\mathscr{A}_{j}^{j}=A_{j}, \mathscr{A}_{1}^{j}=A_{1} A_{2} \cdots A_{j}, \mathscr{X}_{j}^{j}=X_{j}^{*}$ and $\mathscr{X}_{1}^{j}=X_{1}^{*} X_{2}^{*} \cdots X_{j}^{*}$, $j=1,2, \ldots, n$, and define $\mathscr{X}_{n+1}^{n}=I_{l_{n+1}}$. Also, in order to present the necessary and sufficient conditions for the reverse order laws (1.2), (1.3) and the mixed-type reverse order laws (1.4)-(1.7), we define the following two matrix functions:

$$
\begin{aligned}
& T_{A_{1}, A_{2}, \ldots, A_{n}}\left(X_{1}, X_{2}, \ldots, X_{n}\right) \\
= & A_{n}^{*} A_{n-1}^{*} \cdots A_{1}^{*}-A_{n}^{*} A_{n-1}^{*} \cdots A_{1}^{*} A_{1} A_{2} \cdots A_{n} X_{n} X_{n-1} \cdots X_{1}
\end{aligned}
$$

and

$$
\begin{aligned}
& P_{A_{1}, A_{2}, \ldots, A_{n}}\left(X_{1}, X_{2}, \ldots, X_{n}\right) \\
= & A_{1} A_{2} \cdots A_{n}-A_{1} A_{2} \cdots A_{n} X_{n} X_{n-1} \cdots X_{1} A_{1} A_{2} \cdots A_{n} .
\end{aligned}
$$

The main tools in the later discussion are the following three lemmas. The first lemma gives the formulas of the maximal ranks of the generalized Schur complements related to the generalized inverses $[11,12]$, and the second shows the characterizations of some generalized inverses of a matrix.

Lemma 1.1. [11, 12] Let $A \in \mathbb{C}^{m \times n}, B \in \mathbb{C}^{m \times l}, C \in \mathbb{C}^{k \times n}$ and $D \in \mathbb{C}^{k \times l}$. Then

$$
\begin{aligned}
& (1.11) \max _{A^{(1)} \in A\{1\}} r\left(D-C A^{(1)} B\right)=\min \left\{r(C, D), r\left(\begin{array}{c}
B \\
D
\end{array}\right), r\left(\begin{array}{cc}
A & B \\
C & D
\end{array}\right)-r(A)\right\} ; \\
& (1.12) \max _{A^{(1,3)} \in A\{1,3\}} r\left(D-C A^{(1,3)} B\right)=\min \left\{r\left(\begin{array}{lr}
A^{*} A & A^{*} B \\
C & D
\end{array}\right)-r(A), r\left(\begin{array}{c}
B \\
D
\end{array}\right)\right\} .
\end{aligned}
$$

Lemma 1.2. [10] Let $A \in \mathbb{C}^{m \times n}$ and $G \in \mathbb{C}^{n \times m}$. Then

$$
\begin{gathered}
G \in A\{1\} \Leftrightarrow A G A=A ; \\
G \in A\{1,3\} \Leftrightarrow A^{*} A G=A^{*} ; \\
G \in A\{1,4\} \Leftrightarrow G A A^{*}=A^{*} .
\end{gathered}
$$


Lemma 1.3. [20] Let $A_{i} \in \mathbb{C}^{l_{i} \times l_{i+1}}, i=1,2, \ldots, n$. Then

$$
l_{2}+l_{3}+\cdots+l_{n}+r\left(A_{1} \cdots A_{n}\right) \geq r\left(A_{1}\right)+r\left(A_{2}\right)+\cdots+r\left(A_{n}\right) .
$$

In addition, the following rank equalities [7] will be needed in the sequel:

$$
\begin{gathered}
r(A, \quad B)=r(A)+r\left(E_{A} B\right)=r(B)+r\left(E_{B} A\right), \\
r\left(\begin{array}{c}
A \\
C
\end{array}\right)=r(A)+r\left(C F_{A}\right)=r(C)+r\left(A F_{C}\right),
\end{gathered}
$$

where $E_{A}=I-A A^{\dagger}$ and $F_{A}=I-A^{\dagger} A$.

This paper is organized as follows. The necessary and sufficient conditions for the reverse order laws (1.2) and (1.3) to hold are investigated in Section 2. In Section 3 we study the necessary and sufficient conditions for the mixed-type reverse order laws (1.4) and (1.5). Finally, the necessary and sufficient conditions for the mixed-type reverse order laws (1.6) and (1.7) are discussed in Section 4.

2. The necessary and sufficient conditions for the inclusions (1.2) and (1.3). Let $T_{A_{1}, A_{2}, \ldots, A_{n}}\left(X_{1}, X_{2}, \ldots, X_{n}\right)$ be as in (1.9). It is easy to see from the characterization (1.14) of $\{1,3\}$-inverses that the reverse order law (1.2) holds if and only if the following rank identity

$$
\max _{X_{n}, X_{n-1}, \ldots, X_{1}} r\left(T_{A_{1}, A_{2}, \ldots, A_{n}}\left(X_{1}, X_{2}, \ldots, X_{n}\right)\right)=0
$$

holds for any $X_{i} \in A_{i}\{1,3\}, i=1,2, \ldots, n$. Hence, to give the necessary and sufficient conditions for the inclusion (1.2), we first state the following theorem:

Theorem 2.1. Let $A_{i} \in \mathbb{C}^{l_{i} \times l_{i+1}}, i=1,2, \ldots, n, \mathscr{A}_{i}^{j}, 1 \leq i \leq j \leq n$, be as in (1.8) and $T_{A_{1}, A_{2}, \ldots, A_{n}}\left(X_{1}, X_{2}, \ldots, X_{n}\right)$ be as in (1.9). Then

$$
\begin{aligned}
\max _{X_{n}, X_{n-1}, \ldots, X_{1}} r\left(T_{A_{1}, A_{2}, \ldots, A_{n}}\left(X_{1}, X_{2}, \ldots, X_{n}\right)\right) & =\left(\begin{array}{cccc}
A_{n}^{*} & O & \ldots & O \\
O & A_{n-1}^{*} & \cdots & O \\
\vdots & \vdots & \ddots & \vdots \\
O & O & \cdots & A_{2}^{*} \\
\left(\mathscr{A}_{1}^{n}\right)^{*} \mathscr{A}_{1}^{n-1} & \left(\mathscr{A}_{1}^{n}\right)^{*} \mathscr{A}_{1}^{n-2} & \cdots & \left(\mathscr{A}_{1}^{n}\right)^{*} \mathscr{A}_{1}^{1}
\end{array}\right)-\sum_{i=2}^{n} r\left(A_{i}\right),
\end{aligned}
$$

where $X_{i}$ varies over $A_{i}\{1,3\}, i=1,2, \ldots, n$. 
Proof. Let $\mathscr{A}_{i}^{j}, \mathscr{X}_{i}^{j}, 1 \leq i \leq j \leq n$, be as in (1.8). Then according to Lemma 1.1 (1.12) with $A=A_{1}, B=I_{l_{1}}, D=\left(\mathscr{A}_{1}^{n}\right)^{*}$ and $C=\left(\mathscr{A}_{1}^{n}\right)^{*} \mathscr{A}_{1}^{n}\left(\mathscr{X}_{2}^{n}\right)^{*}$, we have

$$
\begin{aligned}
& \max _{X_{1}} r\left(T_{A_{1}, A_{2}, \ldots, A_{n}}\left(X_{1}, X_{2}, \ldots, X_{n}\right)\right) \\
= & \min \left\{r\left(\begin{array}{cc}
A_{1}^{*} A_{1} & A_{1}^{*} \\
\left(\mathscr{A}_{1}^{n}\right)^{*} \mathscr{A}_{1}^{n}\left(\mathscr{X}_{2}^{n}\right)^{*} & \left(\mathscr{A}_{1}^{n}\right)^{*}
\end{array}\right)-r\left(A_{1}\right), r\left(\begin{array}{c}
I_{l_{1}} \\
\left(\mathscr{A}_{1}^{n}\right)^{*}
\end{array}\right)\right\} \\
= & \min \left\{r\left(\left(\mathscr{A}_{1}^{n}\right)^{*} \mathscr{A}_{1}^{n}\left(\mathscr{X}_{2}^{n}\right)^{*}-\left(\mathscr{A}_{1}^{n}\right)^{*} A_{1}\right), l_{1}\right\} \\
= & r\left(\left(\mathscr{A}_{1}^{n}\right)^{*} \mathscr{A}_{1}^{n}\left(\mathscr{X}_{2}^{n}\right)^{*}-\left(\mathscr{A}_{1}^{n}\right)^{*} A_{1}\right),
\end{aligned}
$$

in which, by the row or column elementary block operations, from first equality to second one we use the rank identities

$$
r\left(\begin{array}{c}
I_{l_{1}} \\
\left(\mathscr{A}_{1}^{n}\right)^{*}
\end{array}\right)=l_{1}
$$

and

$$
\begin{aligned}
r\left(\begin{array}{cc}
A_{1}^{*} A_{1} & A_{1}^{*} \\
\left(\mathscr{A}_{1}^{n}\right)^{*} \mathscr{A}_{1}^{n}\left(\mathscr{X}_{2}^{n}\right)^{*} & \left(\mathscr{A}_{1}^{n}\right)^{*}
\end{array}\right) & =r\left(\begin{array}{cc}
0 & A_{1}^{*} \\
\left.\mathscr{A}_{1}^{n}\right)^{*} \mathscr{A}_{1}^{n}\left(\mathscr{X}_{2}^{n}\right)^{*}-\left(\mathscr{A}_{1}^{n}\right)^{*} A_{1} & 0
\end{array}\right) \\
& =r\left(\left(\mathscr{A}_{1}^{n}\right)^{*} \mathscr{A}_{1}^{n}\left(\mathscr{X}_{2}^{n}\right)^{*}-\left(\mathscr{A}_{1}^{n}\right)^{*} A_{1}\right)-r\left(A_{1}\right) .
\end{aligned}
$$

It's worthy mentioning that similar techniques will be repeatedly used in the sequel.

Again by Lemma 1.1 (1.12) with $A=A_{2}, B=I_{l_{2}}, C=\left(\mathscr{A}_{1}^{n}\right)^{*} \mathscr{A}_{1}^{n}\left(\mathscr{X}_{3}^{n}\right)^{*}$ and $D=\left(\mathscr{A}_{1}^{n}\right)^{*} A_{1}$, we have

$$
\begin{aligned}
& \max _{X_{2}, X_{1}} r\left(T_{A_{1}, A_{2}, \ldots, A_{n}}\left(X_{1}, X_{2}, \ldots, X_{n}\right)\right) \\
= & \max _{X_{2}} r\left(\left(\mathscr{A}_{1}^{n}\right)^{*} \mathscr{A}_{1}^{n}\left(\mathscr{X}_{2}^{n}\right)^{*}-\left(\mathscr{A}_{1}^{n}\right)^{*} A_{1}\right) \\
= & \min \left\{r\left(\begin{array}{cc}
A_{2}^{*} A_{2} & A_{2}^{*} \\
\left(\mathscr{A}_{1}^{n}\right)^{*} \mathscr{A}_{1}^{n}\left(\mathscr{X}_{3}^{n}\right)^{*} & \left(\mathscr{A}_{1}^{n}\right)^{*} A_{1}
\end{array}\right)-r\left(A_{2}\right), \quad r\left(\begin{array}{c}
I_{l_{2}} \\
\left(\mathscr{A}_{1}^{n}\right)^{*} A_{1}
\end{array}\right)\right\} \\
= & \min \left\{r\left(\begin{array}{cc}
A_{2}^{*} \\
\left(\mathscr{A}_{1}^{n}\right)^{*} \mathscr{A}_{1}^{n}\left(\mathscr{X}_{3}^{n}\right)^{*}-\left(\mathscr{A}_{1}^{n}\right)^{*} \mathscr{A}_{1}^{2} & \left(\mathscr{A}_{1}^{n}\right)^{*} A_{1}
\end{array}\right)-r\left(A_{2}\right), l_{2}\right\} .
\end{aligned}
$$

By the formula (1.18), we obtain

$$
\begin{aligned}
& r\left(\begin{array}{cc}
O & A_{2}^{*} \\
\left(\mathscr{A}_{1}^{n}\right)^{*} \mathscr{A}_{1}^{n}\left(\mathscr{X}_{3}^{n}\right)^{*}-\left(\mathscr{A}_{1}^{n}\right)^{*} \mathscr{A}_{1}^{2} & \left(\mathscr{A}_{1}^{n}\right)^{*} A_{1}
\end{array}\right) \\
& =r\left(\left(\mathscr{A}_{1}^{n}\right)^{*} \mathscr{A}_{1}^{n}\left(\mathscr{X}_{3}^{n}\right)^{*}-\left(\mathscr{A}_{1}^{n}\right)^{*} \mathscr{A}_{1}^{2}, \quad\left(\mathscr{A}_{1}^{n}\right)^{*} A_{1} F_{A_{2}^{*}}\right)+r\left(A_{2}\right) \\
& =r\left(\left(\mathscr{A}_{1}^{n}\right)^{*} \mathscr{A}_{1}^{1}\left(A_{2} \cdots A_{n}\left(\mathscr{X}_{3}^{n}\right)^{*}-A_{2}\right), \quad\left(\mathscr{A}_{1}^{n}\right)^{*} \mathscr{A}_{1}^{1} F_{A_{2}^{*}}\right)+r\left(A_{2}\right) \\
& \leq r\left(A_{1}\right)+r\left(A_{2}\right) \\
& \leq l_{2}+r\left(A_{2}\right) \text {. }
\end{aligned}
$$


Thus, from (2.4) and (2.5), we have

$$
\begin{aligned}
& \max _{X_{2}, X_{1}} r\left(T_{A_{1}, A_{2}, \ldots, A_{n}}\left(X_{1}, X_{2}, \ldots, X_{n}\right)\right) \\
= & r\left(\left(\mathscr{A}_{1}^{n}\right)^{*} \mathscr{A}_{1}^{n}\left(\mathscr{X}_{3}^{n}\right)^{*}-\left(\mathscr{A}_{1}^{n}\right)^{*} \mathscr{A}_{1}^{2},\left(\mathscr{A}_{1}^{n}\right)^{*} \mathscr{A}_{1}^{1} F_{A_{2}^{*}}\right) .
\end{aligned}
$$

Generally, for $2 \leq i \leq n$, we can prove the following fact:

$$
\begin{aligned}
& \max _{X_{i}, X_{i-1}, \ldots, X_{1}} r\left(T_{A_{1}, A_{2}, \ldots, A_{n}}\left(X_{1}, X_{2}, \ldots, X_{n}\right)\right) \\
& =r\left(\left(\mathscr{A}_{1}^{n}\right)^{*} \mathscr{A}_{1}^{n}\left(\mathscr{X}_{i+1}^{n}\right)^{*}-\left(\mathscr{A}_{1}^{n}\right)^{*} \mathscr{A}_{1}^{i}, \quad\left(\mathscr{A}_{1}^{n}\right)^{*} \mathscr{A}_{1}^{i-1} F_{A_{i}^{*}}, \quad \ldots, \quad\left(\mathscr{A}_{1}^{n}\right)^{*} \mathscr{A}_{1}^{1} F_{A_{2}^{*}}\right) .
\end{aligned}
$$

In fact, the identity (2.7) is true for $i=2$ (see (2.6)). Now assume identity (2.7) is also true for $i-1(i \geq 3)$, that is,

$$
\begin{aligned}
& \text { 2.8) } \max _{X_{i-1}, X_{i-2}, \ldots, X_{1}} r\left(T_{A_{1}, A_{2}, \cdots, A_{n}}\left(X_{1}, X_{2}, \ldots, X_{n}\right)\right) \\
& =r\left(\left(\mathscr{A}_{1}^{n}\right)^{*} \mathscr{A}_{1}^{n}\left(\mathscr{X}_{i}^{n}\right)^{*}-\left(\mathscr{A}_{1}^{n}\right)^{*} \mathscr{A}_{1}^{i-1}, \quad\left(\mathscr{A}_{1}^{n}\right)^{*} \mathscr{A}_{1}^{i-2} F_{A_{i-1}^{*}}, \ldots, \quad\left(\mathscr{A}_{1}^{n}\right)^{*} \mathscr{A}_{1}^{1} F_{A_{2}^{*}}\right) .
\end{aligned}
$$

Next we shall prove that (2.7) is also true for $i$. Combining (2.8) with the formula (1.12) in Lemma 1.1 (with $A=A_{i}, B=\left(I_{l_{i}}, O, \cdots, O\right), C=\left(\mathscr{A}_{1}^{n}\right)^{*} \mathscr{A}_{1}^{n}\left(\mathscr{X}_{i+1}^{n}\right)^{*}$ and $D=\left(\left(\mathscr{A}_{1}^{n}\right)^{*} \mathscr{A}_{1}^{i-1}, \quad\left(\mathscr{A}_{1}^{n}\right)^{*} \mathscr{A}_{1}^{i-2} F_{A_{i-1}^{*}}, \quad \ldots, \quad\left(\mathscr{A}_{1}^{n}\right)^{*} \mathscr{A}_{1}^{1} F_{A_{2}^{*}}\right)$, we have

$$
\begin{aligned}
& \max _{X_{i}, X_{i-1}, \ldots, X_{1}} r\left(T_{A_{1}, A_{2}, \ldots, A_{n}}\left(X_{1}, X_{2}, \ldots, X_{n}\right)\right) \\
& =\max _{X_{i}} r\left(\left(\mathscr{A}_{1}^{n}\right)^{*} \mathscr{A}_{1}^{n}\left(\mathscr{X}_{i}^{n}\right)^{*}-\left(\mathscr{A}_{1}^{n}\right)^{*} \mathscr{A}_{1}^{i-1}, \quad\left(\mathscr{A}_{1}^{n}\right)^{*} \mathscr{A}_{1}^{i-2} F_{A_{i-1}^{*}}, \ldots,\left(\mathscr{A}_{1}^{n}\right)^{*} \mathscr{A}_{1}^{1} F_{A_{2}^{*}}\right) \\
& =\max _{X_{i}} r\left(\left(\mathscr{A}_{1}^{n}\right)^{*} \mathscr{A}_{1}^{n}\left(\mathscr{X}_{i}^{n}\right)^{*}\left(I_{l_{i}}, \quad O, \cdots, \quad O\right)\right. \\
& \left.-\left(\left(\mathscr{A}_{1}^{n}\right)^{*} \mathscr{A}_{1}^{i-1}, \quad\left(\mathscr{A}_{1}^{n}\right)^{*} \mathscr{A}_{1}^{i-2} F_{A_{i-1}^{*}}, \quad \ldots, \quad\left(\mathscr{A}_{1}^{n}\right)^{*} \mathscr{A}_{1}^{1} F_{A_{2}^{*}}\right)\right) \\
& =\min \left\{r\left(\begin{array}{ccccc}
A_{i}^{*} A_{i} & A_{i}^{*} & O & \cdots & O \\
\left(\mathscr{A}_{1}^{n}\right)^{*} \mathscr{A}_{1}^{n}\left(\mathscr{X}_{i+1}^{n}\right)^{*} & \left(\mathscr{A}_{1}^{n}\right)^{*} \mathscr{A}_{1}^{i-1} & \left(\mathscr{A}_{1}^{n}\right)^{*} \mathscr{A}_{1}^{i-2} F_{A_{i-1}^{*}} & \cdots & \left(\mathscr{A}_{1}^{n}\right)^{*} \mathscr{A}_{1}^{1} F_{A_{2}^{*}}
\end{array}\right)\right. \\
& \left.-r\left(A_{i}\right), \quad r\left(\begin{array}{cccc}
I_{l_{i}} & O & \cdots & O \\
\left(\mathscr{A}_{1}^{n}\right)^{*} \mathscr{A}_{1}^{i-1} & \left(\mathscr{A}_{1}^{n}\right)^{*} \mathscr{A}_{1}^{i-2} F_{A_{i-1}^{*}} & \cdots & \left(\mathscr{A}_{1}^{n}\right)^{*} \mathscr{A}_{1}^{1} F_{A_{2}^{*}}
\end{array}\right)\right\} .
\end{aligned}
$$

According to the formula (1.18), we know

$$
\begin{aligned}
& r\left(\begin{array}{ccccc}
A_{i}^{*} A_{i} & A_{i}^{*} & O & \cdots & O \\
\left(\mathscr{A}_{1}^{n}\right)^{*} \mathscr{A}_{1}^{n}\left(\mathscr{X}_{i+1}^{n}\right)^{*} & \left(\mathscr{A}_{1}^{n}\right)^{*} \mathscr{A}_{1}^{i-1} & \left(\mathscr{A}_{1}^{n}\right)^{*} \mathscr{A}_{1}^{i-2} F_{A_{i-1}^{*}} & \cdots & \left(\mathscr{A}_{1}^{n}\right)^{*} \mathscr{A}_{1}^{1} F_{A_{2}^{*}}
\end{array}\right) \\
& =r\left(\begin{array}{cccc}
O & A_{i}^{*} & \cdots & O \\
\left(\mathscr{A}_{1}^{n}\right)^{*} \mathscr{A}_{1}^{n}\left(\mathscr{X}_{i+1}^{n}\right)^{*}-\left(\mathscr{A}_{1}^{n}\right)^{*} \mathscr{A}_{1}^{i} & \left(\mathscr{A}_{1}^{n}\right)^{*} \mathscr{A}_{1}^{i-1} & \cdots & \left(\mathscr{A}_{1}^{n}\right)^{*} \mathscr{A}_{1}^{1} F_{A_{2}^{*}}
\end{array}\right) \\
& =r\left(A_{i}\right)+r\left(\left(\mathscr{A}_{1}^{n}\right)^{*} \mathscr{A}_{1}^{n}\left(\mathscr{X}_{i+1}^{n}\right)^{*}-\left(\mathscr{A}_{1}^{n}\right)^{*} \mathscr{A}_{1}^{i}, \quad\left(\mathscr{A}_{1}^{n}\right)^{*} \mathscr{A}_{1}^{i-1} F_{A_{i}^{*}}, \ldots,\left(\mathscr{A}_{1}^{n}\right)^{*} \mathscr{A}_{1}^{1} F_{A_{2}^{*}}\right) \\
& \leq r\left(A_{i}\right)+r\left(\left(\mathscr{A}_{1}^{n}\right)^{*} \mathscr{A}_{1}^{n}\left(\mathscr{X}_{i+1}^{n}\right)^{*}-\left(\mathscr{A}_{1}^{n}\right)^{*} \mathscr{A}_{1}^{i}, \quad\left(\mathscr{A}_{1}^{n}\right)^{*} \mathscr{A}_{1}^{i-1} F_{A_{i}^{*}}\right) \\
& +r\left(\left(\mathscr{A}_{1}^{n}\right)^{*} \mathscr{A}_{1}^{i-2} F_{A_{i-1}^{*}}, \quad \ldots, \quad\left(\mathscr{A}_{1}^{n}\right)^{*} \mathscr{A}_{1}^{1} F_{A_{2}^{*}}\right)
\end{aligned}
$$




$$
\begin{aligned}
& \leq r\left(A_{i}\right)+r\left(\left(\mathscr{A}_{1}^{n}\right)^{*} \mathscr{A}_{1}^{i-1}\right)+r\left(\left(\mathscr{A}_{1}^{n}\right)^{*} \mathscr{A}_{1}^{i-2} F_{A_{i-1}^{*}}, \quad \ldots, \quad\left(\mathscr{A}_{1}^{n}\right)^{*} \mathscr{A}_{1}^{1} F_{A_{2}^{*}}\right) \\
& \leq r\left(A_{i}\right)+r\left(A_{i-1}\right)+r\left(\left(\mathscr{A}_{1}^{n}\right)^{*} \mathscr{A}_{1}^{i-2} F_{A_{i-1}^{*}}, \quad \ldots, \quad\left(\mathscr{A}_{1}^{n}\right)^{*} \mathscr{A}_{1}^{1} F_{A_{2}^{*}}\right) \\
& \leq r\left(A_{i}\right)+l_{i}+r\left(\left(\mathscr{A}_{1}^{n}\right)^{*} \mathscr{A}_{1}^{i-2} F_{A_{i-1}^{*}}, \quad \ldots, \quad\left(\mathscr{A}_{1}^{n}\right)^{*} \mathscr{A}_{1}^{1} F_{A_{2}^{*}}\right) \text {. }
\end{aligned}
$$

Hence, by (2.9), (2.10) and the formula (1.18) we have

$$
\begin{aligned}
& \max _{X_{i}, X_{i-1}, \ldots, X_{1}} r\left(T_{A_{1}, A_{2}, \ldots, A_{n}}\left(X_{1}, X_{2}, \ldots, X_{n}\right)\right) \\
& =\max _{X_{i}} r\left(\left(\mathscr{A}_{1}^{n}\right)^{*} \mathscr{A}_{1}^{i-1}-\left(\mathscr{A}_{1}^{n}\right)^{*} \mathscr{A}_{1}^{n}\left(\mathscr{X}_{i}^{n}\right)^{*}, \quad\left(\mathscr{A}_{1}^{n}\right)^{*} \mathscr{A}_{1}^{i-2} F_{A_{i-1}^{*}}, \ldots,\left(\mathscr{A}_{1}^{n}\right)^{*} \mathscr{A}_{1}^{1} F_{A_{2}^{*}}\right) \\
& =r\left(\begin{array}{ccccc}
A_{i}^{*} A_{i} & A_{i}^{*} & O & \cdots & O \\
\left(\mathscr{A}_{1}^{n}\right)^{*} \mathscr{A}_{1}^{n}\left(\mathscr{X}_{i+1}^{n}\right)^{*} & \left(\mathscr{A}_{1}^{n}\right)^{*} \mathscr{A}_{1}^{i-1} & \left(\mathscr{A}_{1}^{n}\right)^{*} \mathscr{A}_{1}^{i-2} F_{A_{i-1}^{*}} & \cdots & \left(\mathscr{A}_{1}^{n}\right)^{*} \mathscr{A}_{1}^{1} F_{A_{2}^{*}}
\end{array}\right)-r\left(A_{i}\right) \\
& =r\left(\left(\mathscr{A}_{1}^{n}\right)^{*} \mathscr{A}_{1}^{n}\left(\mathscr{X}_{i+1}^{n}\right)^{*}-\left(\mathscr{A}_{1}^{n}\right)^{*} \mathscr{A}_{1}^{i}, \quad\left(\mathscr{A}_{1}^{n}\right)^{*} \mathscr{A}_{1}^{i-1} F_{A_{i}^{*}}, \quad \ldots, \quad\left(\mathscr{A}_{1}^{n}\right)^{*} \mathscr{A}_{1}^{1} F_{A_{2}^{*}}\right) .
\end{aligned}
$$

Specifically, in the case of $i=n$, we obtain

$$
\begin{aligned}
& \max _{X_{n}, X_{n-1}, \ldots, X_{1}} r\left(T_{A_{1}, A_{2}, \ldots, A_{n}}\left(X_{1}, X_{2}, \ldots, X_{n}\right)\right) \\
= & r\left(\left(\mathscr{A}_{1}^{n}\right)^{*} \mathscr{A}_{1}^{n}\left(\mathscr{X}_{n+1}^{n}\right)^{*}-\left(\mathscr{A}_{1}^{n}\right)^{*} \mathscr{A}_{1}^{n}, \quad\left(\mathscr{A}_{1}^{n}\right)^{*} \mathscr{A}_{1}^{n-1} F_{A_{n}^{*}}, \quad \ldots, \quad\left(\mathscr{A}_{1}^{n}\right)^{*} \mathscr{A}_{1}^{1} F_{A_{2}^{*}}\right) \\
= & r\left(\left(\mathscr{A}_{1}^{n}\right)^{*} \mathscr{A}_{1}^{n}-\left(\mathscr{A}_{1}^{n}\right)^{*} \mathscr{A}_{1}^{n}, \quad\left(\mathscr{A}_{1}^{n}\right)^{*} \mathscr{A}_{1}^{n-1} F_{A_{n}^{*}}, \ldots, \quad \ldots, \quad\left(\mathscr{A}_{1}^{n}\right)^{*} \mathscr{A}_{1}^{1} F_{A_{2}^{*}}\right) \\
= & r\left(\left(\mathscr{A}_{1}^{n}\right)^{*} \mathscr{A}_{1}^{n-1} F_{A_{n}^{*}}, \quad\left(\mathscr{A}_{1}^{n}\right)^{*} \mathscr{A}_{1}^{n-2} F_{A_{n-1}^{*}}, \quad \ldots, \quad\left(\mathscr{A}_{1}^{n}\right)^{*} \mathscr{A}_{1}^{1} F_{A_{2}^{*}}\right) .
\end{aligned}
$$

We now repeatedly apply formula (1.18) to (2.11) and finally have

$$
\begin{aligned}
& \max _{X_{n}, X_{n-1}, \ldots, X_{1}} r\left(T_{A_{1}, A_{2}, \ldots, A_{n}}\left(X_{1}, X_{2}, \ldots, X_{n}\right)\right) \\
& =r\left(\left(\mathscr{A}_{1}^{n}\right)^{*} \mathscr{A}_{1}^{n-1} F_{A_{n}^{*}}, \quad\left(\mathscr{A}_{1}^{n}\right)^{*} \mathscr{A}_{1}^{n-2} F_{A_{n-1}^{*}}, \quad \ldots, \quad\left(\mathscr{A}_{1}^{n}\right)^{*} \mathscr{A}_{1}^{1} F_{A_{2}^{*}}\right) \\
& =r\left(\begin{array}{cccc}
A_{n}^{*} & O & \cdots & O \\
O & A_{n-1}^{*} & \cdots & O \\
\vdots & \vdots & \ddots & \vdots \\
O & O & \cdots & A_{2}^{*} \\
\left(\mathscr{A}_{1}^{n}\right)^{*} \mathscr{A}_{1}^{n-1} & \left(\mathscr{A}_{1}^{n}\right)^{*} \mathscr{A}_{1}^{n-2} & \cdots & \left(\mathscr{A}_{1}^{n}\right)^{*} \mathscr{A}_{1}^{1}
\end{array}\right)-\sum_{i=2}^{n} r\left(A_{i}\right) .
\end{aligned}
$$

From Theorem 2.1 and the identity (2.1), we can immediately get the necessary and sufficient conditions for the inclusion (1.2) to hold, which are stated in the following theorem.

TheOrEm 2.2. Let $A_{i} \in \mathbb{C}^{l_{i} \times l_{i+1}}, i=1,2, \ldots, n$, and $\mathscr{A}_{i}^{j}, 1 \leq i \leq j \leq n$, be as in (1.8). Then the following statements are equivalent:

(1) $A_{n}\{1,3\} A_{n-1}\{1,3\} \cdots A_{1}\{1,3\} \subseteq\left(A_{1} A_{2} \ldots A_{n}\right)\{1,3\}$;

(2) $\quad r\left(\left(\mathscr{A}_{1}^{n}\right)^{*} \mathscr{A}_{1}^{n-1} F_{A_{n}^{*}}, \quad\left(\mathscr{A}_{1}^{n}\right)^{*} \mathscr{A}_{1}^{n-2} F_{A_{n-1}^{*}}, \quad \ldots, \quad\left(\mathscr{A}_{1}^{n}\right)^{*} \mathscr{A}_{1}^{1} F_{A_{2}^{*}}\right)=0$; 
(3) $R\left(\left(\mathscr{A}_{1}^{i}\right)^{*} \mathscr{A}_{1}^{n}\right) \subseteq R\left(A_{i+1}\right), \quad i=1,2, \ldots, n-1$;

(4) $r\left(\begin{array}{cccc}A_{n}^{*} & O & \cdots & O \\ O & A_{n-1}^{*} & \cdots & O \\ \vdots & \vdots & \ddots & \vdots \\ O & O & \cdots & A_{2}^{*} \\ \left(\mathscr{A}_{1}^{n}\right)^{*} \mathscr{A}_{1}^{n-1} & \left(\mathscr{A}_{1}^{n}\right)^{*} \mathscr{A}_{1}^{n-2} & \cdots & \left(\mathscr{A}_{1}^{n}\right)^{*} \mathscr{A}_{1}^{1}\end{array}\right)=\sum_{i=2}^{n} r\left(A_{i}\right)$.

Before presenting an example to confirm Theorem 2.2, we mention the following general expressions of $\{1,3\}-$ and $\{1,4\}$-inverse for any matrix $A \in \mathbb{C}^{m \times n}$ :

$$
\begin{aligned}
& A\{1,3\}=\left\{A^{\dagger}+\left(I_{n}-A^{\dagger} A\right) X: X \in \mathbb{C}^{n \times m}\right\}, \\
& A\{1,4\}=\left\{A^{\dagger}+Y\left(I_{m}-A A^{\dagger}\right): Y \in \mathbb{C}^{n \times m}\right\} .
\end{aligned}
$$

If we know the Moore-Penrose inverse $A^{\dagger}$ of matrix $A$, then the above formulas make very easy the computations of $A\{1,3\}$ and $A\{1,4\}$.

EXAMPLE 2.3. Let

$$
A_{1}=\left(\begin{array}{ll}
1 & 0 \\
1 & 0
\end{array}\right), A_{2}=\left(\begin{array}{lll}
1 & 0 & 0 \\
1 & 1 & 0
\end{array}\right) \text { and } A_{3}=\left(\begin{array}{lll}
1 & 0 & 0 \\
0 & 1 & 1 \\
0 & 1 & 1
\end{array}\right)
$$

Then

$$
r\left(\begin{array}{cc}
A_{3}^{*} & O \\
O & A_{2}^{*} \\
A_{3}^{*} A_{2}^{*} A_{1}^{*} A_{1} A_{2} & A_{3}^{*} A_{2}^{*} A_{1}^{*} A_{1}
\end{array}\right)=r\left(A_{2}\right)+r\left(A_{3}\right)=4,
$$

which means that the matrices $A_{1}, A_{2}$ and $A_{3}$ satisfy the condition (4) in Theorem 2.2. On the other hand, by the formula (2.13), we have

$$
A_{1}\{1,3\}=\left\{\left(\begin{array}{cc}
1 / 2 & 1 / 2 \\
a_{1} & b_{1}
\end{array}\right) \mid a_{1}, b_{1} \in \mathbb{C}\right\}, \quad A_{2}\{1,3\}=\left\{\left(\begin{array}{cc}
1 & 0 \\
-1 & 1 \\
a_{2} & b_{2}
\end{array}\right) \mid a_{2}, b_{2} \in \mathbb{C}\right\}
$$

and

$$
A_{3}\{1,3\}=\left\{\left(\begin{array}{ccc}
1 & 0 & 0 \\
a_{3} & b_{3} & c \\
-a_{3} & 1 / 2-b_{3} & 1 / 2-c
\end{array}\right) \mid a_{3}, b_{3}, c \in \mathbb{C}\right\} .
$$

Hence, the matrix set $A_{3}\{1,3\} A_{2}\{1,3\} A_{1}\{1,3\}$ can be expressed as

$$
\begin{aligned}
& A_{3}\{1,3\} A_{2}\{1,3\} A_{1}\{1,3\}=\left\{M_{1}:\right. \\
& \left.M_{1}=\left(\begin{array}{ccc}
1 & 0 & 0 \\
a_{3} & b_{3} & c \\
-a_{3} & 1 / 2-b_{3} & 1 / 2-c
\end{array}\right)\left(\begin{array}{cc}
1 & 0 \\
-1 & 1 \\
a_{2} & b_{2}
\end{array}\right)\left(\begin{array}{cc}
1 / 2 & 1 / 2 \\
a_{1} & b_{1}
\end{array}\right) \mid a_{i}, b_{j}, c \in \mathbb{C}\right\} .
\end{aligned}
$$


It is easy to verify that the identities

$$
\left(A_{1} A_{2} A_{3}\right) M_{1}\left(A_{1} A_{2} A_{3}\right)=A_{1} A_{2} A_{3} \text { and }\left(A_{1} A_{2} A_{3} M_{1}\right)^{*}=A_{1} A_{2} A_{3} M_{1}
$$

hold for any matrix $M_{1} \in A_{3}\{1,3\} A_{2}\{1,3\} A_{1}\{1,3\}$, that is

$$
A_{3}\{1,3\} A_{2}\{1,3\} A_{1}\{1,3\} \subseteq\left(A_{1} A_{2} A_{3}\right)\{1,3\} .
$$

In the remainder of this section, we will present the necessary and sufficient conditions for the inclusion (1.3) involved $\{1,4\}$-inverses. Notice that $G A A^{*}=A^{*}$ is equivalent to the equation $A A^{*} G^{*}=A$. This implies that, by Lemma $1.2, G \in A\{1,4\}$ if and only if $G^{*} \in A^{*}\{1,3\}$. So from the results obtained in Theorem 2.2 , we can immediately get the necessary and sufficient conditions for the inclusion (1.3), which are stated below without proofs.

Theorem 2.4. Let $A_{i} \in \mathbb{C}^{l_{i} \times l_{i+1}}, i=1,2, \ldots, n$, and $\mathscr{A}_{i}^{j}, 1 \leq i \leq j \leq n$, be as in (1.8). Then the following statements are equivalent:

(1) $A_{n}\{1,4\} A_{n-1}\{1,4\} \cdots A_{1}\{1,4\} \subseteq\left(A_{1} A_{2} \cdots A_{n}\right)\{1,4\}$;

(2) $r\left(\begin{array}{c}E_{A_{1}^{*}} \mathscr{A}_{2}^{n}\left(\mathscr{A}_{1}^{n}\right)^{*} \\ E_{A_{2}^{*}} \mathscr{A}_{3}^{n}\left(\mathscr{A}_{1}^{n}\right)^{*} \\ \vdots \\ E_{A_{n-1}^{*}} \mathscr{A}_{n}^{n}\left(\mathscr{A}_{1}^{n}\right)^{*}\end{array}\right)=0$

(3) $R\left(\mathscr{A}_{i}^{n}\left(\mathscr{A}_{1}^{n}\right)^{*}\right) \subseteq R\left(A_{i-1}^{*}\right), \quad i=2,3, \ldots, n$;

(4) $r\left(\begin{array}{ccccc}A_{1}^{*} & O & \cdots & O & \mathscr{A}_{2}^{n}\left(\mathscr{A}_{1}^{n}\right)^{*} \\ O & A_{2}^{*} & \cdots & O & \mathscr{A}_{3}^{n}\left(\mathscr{A}_{1}^{n}\right)^{*} \\ \vdots & \vdots & \ddots & \vdots & \vdots \\ O & O & \cdots & A_{n-1}^{*} & \mathscr{A}_{n}^{n}\left(\mathscr{A}_{1}^{n}\right)^{*}\end{array}\right)=\sum_{i=1}^{n-1} r\left(A_{i}\right)$.

Similar to Example 2.3, we can easily verify the following three matrices

$$
A_{1}=\left(\begin{array}{lll}
1 & 1 & 1 \\
0 & 1 & 1 \\
0 & 1 & 1
\end{array}\right), A_{2}=\left(\begin{array}{ll}
1 & 0 \\
1 & 1 \\
1 & 1
\end{array}\right) \text { and } A_{3}=\left(\begin{array}{ll}
1 & 1 \\
1 & 1
\end{array}\right)
$$

satisfy the inclusion relationship

$$
A_{3}\{1,4\} A_{2}\{1,4\} A_{1}\{1,4\} \subseteq\left(A_{1} A_{2} A_{3}\right)\{1,4\} .
$$

3. The necessary and sufficient conditions for the inclusions (1.4) and (1.5). Consider the matrix function $P_{A_{1}, A_{2}, \ldots, A_{n}}\left(X_{1}, X_{2}, \ldots, X_{n}\right)$ defined in (1.10). 
By Lemma 1.2 (1.13), we know that the mixed-type reverse order law (1.4) holds if and only if the rank identity

$$
\max _{X_{n}, X_{n-1}, \ldots, X_{1}} r\left(P_{A_{1}, A_{2}, \ldots, A_{n}}\left(X_{1}, X_{2}, \ldots, X_{n}\right)\right)=0
$$

holds, where each $X_{i}(i=1,2, \ldots, n)$ varies over the set $A_{i}\{1,3\}$ of all $\{1,3\}$-inverses of the matrix $A_{i}$. Hence, to give the necessary and sufficient conditions for the inclusion (1.4), we first study the concrete expression of the maximum rank of the matrix function $P_{A_{1}, A_{2}, \ldots, A_{n}}\left(X_{1}, X_{2}, \ldots, X_{n}\right)$ when $X_{i} \in A_{i}\{1,3\}, i=1,2, \ldots, n$.

Theorem 3.1. Let $A_{i} \in \mathbb{C}^{l_{i} \times l_{i+1}}, i=1, \ldots, n$, and $P_{A_{1}, A_{2}, \ldots, A_{n}}\left(X_{1}, X_{2}, \ldots, X_{n}\right)$ be as in (1.10). Then

$$
\begin{aligned}
& \max _{X_{n}, X_{n-1}, \ldots, X_{1}} r\left(P_{A_{1}, A_{2}, \ldots, A_{n}}\left(X_{1}, X_{2}, \ldots, X_{n}\right)\right) \\
& =r\left(\begin{array}{cccc}
A_{n}^{*} & O & \cdots & O \\
O & A_{n-1}^{*} & \cdots & O \\
\vdots & \vdots & \ddots & \vdots \\
O & O & \cdots & A_{2}^{*} \\
A_{1} A_{2} \cdots A_{n-1} & A_{1} A_{2} \cdots A_{n-2} & \cdots & A_{1}
\end{array}\right)+r\left(A_{1} A_{2} \cdots A_{n}\right)-\sum_{i=1}^{n} r\left(A_{i}\right),
\end{aligned}
$$

where $X_{i}$ varies over $A_{i}\{1,3\}, i=1,2, \ldots, n$.

Proof. The basic idea for the proof of Theorem 3.1 is similar to that of Theorem 2.1. For the completeness of the paper, we still give the detailed proof here.

Let $\mathscr{A}_{i}^{j}, \mathscr{X}_{i}^{j}, 1 \leq i \leq j \leq n$, be as in (1.8). Then, by Lemma 1.1 (1.12) with $A=A_{1}, B=\mathscr{A}_{1}^{n}, D=\mathscr{A}_{1}^{n}$ and $C=\mathscr{A}_{1}^{n}\left(\mathscr{X}_{2}^{n}\right)^{*}$, we have

$$
\begin{aligned}
& \max _{X_{1}} r\left(P_{A_{1}, A_{2}, \ldots, A_{n}}\left(X_{1}, X_{2}, \ldots, X_{n}\right)\right) \\
= & \min \left\{r\left(\begin{array}{cc}
A_{1}^{*} A_{1} & A_{1}^{*} \mathscr{A}_{1}^{n} \\
\mathscr{A}_{1}^{n}\left(\mathscr{X}_{2}^{n}\right)^{*} & \mathscr{A}_{1}^{n}
\end{array}\right)-r\left(A_{1}\right), r\left(\begin{array}{c}
\mathscr{A}_{1}^{n} \\
\mathscr{A}_{1}^{n}
\end{array}\right)\right\} \\
= & \min \left\{r\left(\begin{array}{cc}
A_{1} & \mathscr{A}_{1}^{n} \\
\mathscr{A}_{1}^{n}\left(\mathscr{X}_{2}^{n}\right)^{*} & \mathscr{A}_{1}^{n}
\end{array}\right)-r\left(A_{1}\right), \quad r\left(\mathscr{A}_{1}^{n}\right)\right\} \\
= & \min \left\{r\left(\mathscr{A}_{1}^{n}\left(\mathscr{X}_{2}^{n}\right)^{*}-A_{1}\right)+r\left(A_{1} A_{2} \cdots A_{n}\right)-r\left(A_{1}\right), \quad r\left(A_{1} A_{2} \cdots A_{n}\right)\right\} \\
= & r\left(\mathscr{A}_{1}^{n}\left(\mathscr{X}_{2}^{n}\right)^{*}-A_{1}\right)+r\left(A_{1} A_{2} \cdots A_{n}\right)-r\left(A_{1}\right) .
\end{aligned}
$$

Again by Lemma 1.1 (1.12) with $A=A_{2}, B=I_{l_{2}}, C=\mathscr{A}_{1}^{n}\left(\mathscr{X}_{3}^{n}\right)^{*}$ and $D=A_{1}$, we know that

$$
\begin{aligned}
& \max _{X_{2}} r\left(\mathscr{A}_{1}^{n}\left(\mathscr{X}_{2}^{n}\right)^{*}-A_{1}\right) \\
= & \min \left\{r\left(\begin{array}{cc}
A_{2}^{*} A_{2} & A_{2}^{*} \\
\mathscr{A}_{1}^{n}\left(\mathscr{X}_{3}^{n}\right)^{*} & A_{1}
\end{array}\right)-r\left(A_{2}\right), r\left(\begin{array}{c}
I_{l_{2}} \\
A_{1}
\end{array}\right)\right\}
\end{aligned}
$$




$$
\begin{aligned}
& =\min \left\{r\left(\begin{array}{cc}
O & A_{2}^{*} \\
\mathscr{A}_{1}^{n}\left(\mathscr{X}_{3}^{n}\right)^{*}-\mathscr{A}_{1}^{2} & \mathscr{A}_{1}^{1}
\end{array}\right)-r\left(A_{2}\right), l_{2}\right\} \\
& =\min \left\{r\left(\mathscr{A}_{1}^{n}\left(\mathscr{X}_{3}^{n}\right)^{*}-\mathscr{A}_{1}^{2}, \quad \mathscr{A}_{1}^{1} F_{A_{2}^{*}}\right)+r\left(A_{2}\right)-r\left(A_{2}\right), l_{2}\right\} \\
& =\min \left\{r\left(A_{1}\left(\mathscr{A}_{2}^{n}\left(\mathscr{X}_{3}^{n}\right)^{*}-A_{2}, \quad F_{A_{2}^{*}}\right)\right), l_{2}\right\} \\
& =r\left(\mathscr{A}_{1}^{n}\left(\mathscr{X}_{3}^{n}\right)^{*}-\mathscr{A}_{1}^{2}, \mathscr{A}_{1}^{1} F_{A_{2}^{*}}\right),
\end{aligned}
$$

where the rank identity (1.18) is used in the third equality. Hence, from (3.3) and (3.4), we have

$$
\begin{aligned}
& \max _{X_{2}, X_{1}} r\left(P_{A_{1}, A_{2}, \ldots, A_{n}}\left(X_{1}, X_{2}, \ldots, X_{n}\right)\right) \\
= & \max _{X_{2}} r\left(\mathscr{A}_{1}^{n}\left(\mathscr{X}_{2}^{n}\right)^{*}-A_{1}\right)+r\left(A_{1} A_{2} \cdots A_{n}\right)-r\left(A_{1}\right) \\
= & r\left(\mathscr{A}_{1}^{n}\left(\mathscr{X}_{3}^{n}\right)^{*}-\mathscr{A}_{1}^{2}, \quad \mathscr{A}_{1}^{1} F_{A_{2}^{*}}\right)+r\left(A_{1} A_{2} \cdots A_{n}\right)-r\left(A_{1}\right) .
\end{aligned}
$$

We assert that, for $2 \leq i \leq n$,

$$
\begin{aligned}
& \max X_{i}, X_{i-1}, \ldots, X_{1} \\
= & r\left(P_{A_{1}, A_{2}, \ldots, A_{n}}\left(X_{1}, X_{2}, \ldots, X_{n}\right)\right) \\
& \left.+r\left(\mathscr{X}_{i+1}^{n}\right)^{*}-\mathscr{A}_{1}^{i}, \quad \mathscr{A}_{1}^{i-1} F_{A_{i}^{*}}, \quad \mathscr{A}_{1}^{i-2} F_{A_{i-1}^{*}}, \cdots, \mathscr{A}_{1}^{1} F_{A_{2}^{*}}\right) \\
&
\end{aligned}
$$

This can be proved by induction on $i$. In fact, the identity (3.5) has shown the truth of the equality relation (3.6) for $i=2$. Assume that (3.6) is true for $i-1(i \geq 3)$, that is

$$
\begin{aligned}
& \max _{X_{i-1}, X_{i-2}, \ldots, X_{1}} r\left(P_{A_{1}, A_{2}, \ldots, A_{n}}\left(X_{1}, X_{2}, \ldots, X_{n}\right)\right) \\
= & r\left(\mathscr{A}_{1}^{n}\left(\mathscr{X}_{i}^{n}\right)^{*}-\mathscr{A}_{1}^{i-1}, \quad \mathscr{A}_{1}^{i-2} F_{A_{i-1}^{*}}, \quad \mathscr{A}_{1}^{i-3} F_{A_{i-2}^{*}}, \cdots, \quad \mathscr{A}_{1}^{1} F_{A_{2}^{*}}\right) \\
& +r\left(A_{1} A_{2} \cdots A_{n}\right)-r\left(A_{1}\right) .
\end{aligned}
$$

We now prove that (3.6) is also true for $i$. Combining (3.7) with the formula (1.12)

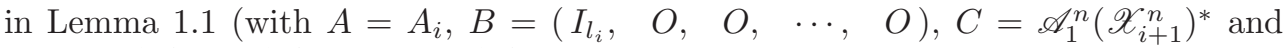
$\left.D=\left(\mathscr{A}_{1}^{i-1}, \quad \mathscr{A}_{1}^{i-2} F_{A_{i-1}^{*}}, \quad \mathscr{A}_{1}^{i-3} F_{A_{i-2}^{*}}, \quad \cdots, \quad \mathscr{A}_{1}^{1} F_{A_{2}^{*}}\right)\right)$, we have

$$
\begin{aligned}
& \text { (3.8) } \max _{X_{i}, X_{i-1}, \ldots, X_{1}} r\left(P_{A_{1}, A_{2}, \ldots, A_{n}}\left(X_{1}, X_{2}, \ldots, X_{n}\right)\right) \\
& =\max _{X_{i}} r\left(\mathscr{A}_{1}^{n}\left(\mathscr{X}_{i}^{n}\right)^{*}-\mathscr{A}_{1}^{i-1}, \quad \mathscr{A}_{1}^{i-2} F_{A_{i-1}^{*}}, \quad \mathscr{A}_{1}^{i-3} F_{A_{i-2}^{*}}, \quad \cdots, \quad \mathscr{A}_{1}^{1} F_{A_{2}^{*}}\right) \\
& +r\left(A_{1} A_{2} \cdots A_{n}\right)-r\left(A_{1}\right) \\
& =\max _{X_{i}} r\left(\mathscr{A}_{1}^{n}\left(\mathscr{X}_{i}^{n}\right)^{*}\left(I_{l_{i}}, \quad O, \quad O, \quad \cdots, \quad O\right)\right. \\
& \left.-\left(\mathscr{A}_{1}^{i-1}, \quad \mathscr{A}_{1}^{i-2} F_{A_{i-1}^{*}}, \quad \mathscr{A}_{1}^{i-3} F_{A_{i-2}^{*}}, \quad \cdots, \quad \mathscr{A}_{1}^{1} F_{A_{2}^{*}}\right)\right)+r\left(A_{1} \cdots A_{n}\right)-r\left(A_{1}\right)
\end{aligned}
$$

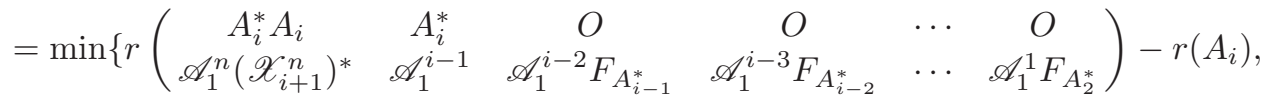


1096

$$
\begin{aligned}
& \left.r\left(\begin{array}{ccccc}
I_{l_{i}} & O & O & \cdots & O \\
\mathscr{A}_{1}^{i-1} & \mathscr{A}_{1}^{i-2} F_{A_{i-1}^{*}} & \mathscr{A}_{1}^{i-3} F_{A_{i-2}^{*}} & \cdots & \mathscr{A}_{1}^{1} F_{A_{2}^{*}}
\end{array}\right)\right\}+r\left(A_{1} A_{2} \cdots A_{n}\right)-r\left(A_{1}\right) \\
& =\min \left\{r\left(\begin{array}{ccccc}
O & A_{i}^{*} & O & \cdots & O \\
\mathscr{A}_{1}^{n}\left(\mathscr{X}_{i+1}^{n}\right)^{*}-\mathscr{A}_{1}^{i} & \mathscr{A}_{1}^{i-1} & \mathscr{A}_{1}^{i-2} F_{A_{i-1}^{*}} & \cdots & \mathscr{A}_{1}^{1} F_{A_{2}^{*}}
\end{array}\right)-r\left(A_{i}\right),\right. \\
& \left.l_{i}+r\left(\mathscr{A}_{1}^{i-2} F_{A_{i-1}^{*}}, \quad \mathscr{A}_{1}^{i-3} F_{A_{i-2}^{*}}, \cdots, \quad \cdots \mathscr{A}_{1}^{1} F_{A_{2}^{*}}\right)\right\}+r\left(A_{1} A_{2} \cdots A_{n}\right)-r\left(A_{1}\right) .
\end{aligned}
$$

Since

$$
\begin{aligned}
& r\left(\begin{array}{cccccc}
O & A_{i}^{*} & O & O & \cdots & O \\
\mathscr{A}_{1}^{n}\left(\mathscr{X}_{i+1}^{n}\right)^{*}-\mathscr{A}_{1}^{i} & \mathscr{A}_{1}^{i-1} & \mathscr{A}_{1}^{i-2} F_{A_{i-1}^{*}} & \mathscr{A}_{1}^{i-3} F_{A_{i-2}^{*}} & \cdots & \mathscr{A}_{1}^{1} F_{A_{2}^{*}}
\end{array}\right) \\
& =r\left(A_{i}\right)+r\left(\mathscr{A}_{1}^{n}\left(\mathscr{X}_{i+1}^{n}\right)^{*}-\mathscr{A}_{1}^{i}, \quad \mathscr{A}_{1}^{i-1} F_{A_{i}^{*}}, \quad \mathscr{A}_{1}^{i-2} F_{A_{i-1}^{*}}, \cdots, \quad \cdots, \mathscr{A}_{1}^{1} F_{A_{2}^{*}}\right) \\
& \leq r\left(A_{i}\right)+r\left(\mathscr{A}_{1}^{n}\left(\mathscr{X}_{i+1}^{n}\right)^{*}-\mathscr{A}_{1}^{i}, \quad \mathscr{A}_{1}^{i-1} F_{A_{i}^{*}}\right) \\
& +r\left(\mathscr{A}_{1}^{i-2} F_{A_{i-1}^{*}}, \quad \mathscr{A}_{1}^{i-3} F_{A_{i-2}^{*}}, \quad \cdots, \quad \mathscr{A}_{1}^{1} F_{A_{2}^{*}}\right) \\
& \leq r\left(A_{i}\right)+r\left(\mathscr{A}_{1}^{i-1}\right)+r\left(\mathscr{A}_{1}^{i-2} F_{A_{i-1}^{*}}, \quad \mathscr{A}_{1}^{i-3} F_{A_{i-2}^{*}}, \quad \cdots, \quad \mathscr{A}_{1}^{1} F_{A_{2}^{*}}\right) \\
& \leq r\left(A_{i}\right)+r\left(A_{i-1}\right)+r\left(\mathscr{A}_{1}^{i-2} F_{A_{i-1}^{*}}, \quad \mathscr{A}_{1}^{i-3} F_{A_{i-2}^{*}}, \quad \cdots, \quad \mathscr{A}_{1}^{1} F_{A_{2}^{*}}\right) \\
& \leq r\left(A_{i}\right)+l_{i}+r\left(\mathscr{A}_{1}^{i-2} F_{A_{i-1}^{*}}, \quad \mathscr{A}_{1}^{i-3} F_{A_{i-2}^{*}}, \quad \cdots, \quad \mathscr{A}_{1}^{1} F_{A_{2}^{*}}\right)
\end{aligned}
$$

in which we have used the identity (1.18) in the first equality, so from (3.8) and (3.9) we have

$$
\begin{aligned}
& \max _{X_{i}, X_{i-1}, \ldots, X_{1}} r\left(P_{A_{1}, A_{2}, \ldots, A_{n}}\left(X_{1}, X_{2}, \ldots, X_{n}\right)\right) \\
= & r\left(\mathscr{A}_{1}^{n}\left(\mathscr{X}_{i+1}^{n}\right)^{*}-\mathscr{A}_{1}^{i}, \quad \mathscr{A}_{1}^{i-1} F_{A_{i}^{*}}, \mathscr{A}_{1}^{i-2} F_{A_{i-1}^{*}}, \cdots, \quad \mathscr{A}_{1}^{1} F_{A_{2}^{*}}\right) \\
& +r\left(A_{1} A_{2} \cdots A_{n}\right)-r\left(A_{1}\right) .
\end{aligned}
$$

In particular, when $i=n$, we have

$$
\begin{aligned}
& \max _{X_{n}, X_{n-1}, \ldots, X_{1}} r\left(P_{A_{1}, A_{2}, \ldots, A_{n}}\left(X_{1}, X_{2}, \ldots, X_{n}\right)\right) \\
& =r\left(\mathscr{A}_{1}^{n}\left(\mathscr{X}_{n+1}^{n}\right)^{*}-\mathscr{A}_{1}^{n}, \quad \mathscr{A}_{1}^{n-1} F_{A_{n}^{*}}, \quad \mathscr{A}_{1}^{n-2} F_{A_{n-1}^{*}}, \quad \cdots, \quad \mathscr{A}_{1}^{1} F_{A_{2}^{*}}\right) \\
& +r\left(A_{1} A_{2} \cdots A_{n}\right)-r\left(A_{1}\right) \\
& =r\left(\mathscr{A}_{1}^{n}-\mathscr{A}_{1}^{n}, \quad \mathscr{A}_{1}^{n-1} F_{A_{n}^{*}}, \quad \mathscr{A}_{1}^{n-2} F_{A_{n-1}^{*}}, \cdots, \quad \mathscr{A}_{1}^{1} F_{A_{2}^{*}}\right) \\
& +r\left(A_{1} A_{2} \cdots A_{n}\right)-r\left(A_{1}\right) \\
& =r\left(\mathscr{A}_{1}^{n-1} F_{A_{n}^{*}}, \quad \mathscr{A}_{1}^{n-2} F_{A_{n-1}^{*}}, \quad \cdots, \quad \mathscr{A}_{1}^{1} F_{A_{2}^{*}}\right)+r\left(A_{1} A_{2} \cdots A_{n}\right)-r\left(A_{1}\right) .
\end{aligned}
$$

By repeatedly applying the formula (1.18) to (3.10), we can finally have

$$
\begin{aligned}
& \max _{X_{n}, X_{n-1}, \ldots, X_{1}} r\left(P_{A_{1}, A_{2}, \ldots, A_{n}}\left(X_{1}, X_{2}, \ldots, X_{n}\right)\right) \\
& =r\left(\mathscr{A}_{1}^{n-1} F_{A_{n}^{*}}, \quad \mathscr{A}_{1}^{n-2} F_{A_{n-1}^{*}}, \quad \cdots, \quad \mathscr{A}_{1}^{1} F_{A_{2}^{*}}\right)+r\left(A_{1} A_{2} \cdots A_{n}\right)-r\left(A_{1}\right) \\
& =r\left(\begin{array}{cccc}
A_{n}^{*} & O & \cdots & O \\
O & A_{n-1}^{*} & \cdots & O \\
\vdots & \vdots & \ddots & \vdots \\
O & O & \cdots & A_{2}^{*} \\
A_{1} \cdots A_{n-1} & A_{1} \cdots A_{n-2} & \cdots & A_{1}
\end{array}\right)-\sum_{i=2}^{n} r\left(A_{i}\right)+r\left(A_{1} A_{2} \cdots A_{n}\right)-r\left(A_{1}\right)
\end{aligned}
$$




$$
=r\left(\begin{array}{cccc}
A_{n}^{*} & O & \cdots & O \\
O & A_{n-1}^{*} & \cdots & O \\
\vdots & \vdots & \ddots & \vdots \\
O & O & \cdots & A_{2}^{*} \\
A_{1} A_{2} \cdots A_{n-1} & A_{1} A_{2} \cdots A_{n-2} & \cdots & A_{1}
\end{array}\right)+r\left(A_{1} A_{2} \cdots A_{n}\right)-\sum_{i=1}^{n} r\left(A_{i}\right) .
$$

The next theorem is a direct result from Theorem 3.1 and the identity (3.1). It provides some equivalently necessary and sufficient conditions for the inclusion (1.4) to hold.

TheOREM 3.2. Let $A_{i} \in \mathbb{C}^{l_{i} \times l_{i+1}}, i=1,2, \ldots, n$. Then the following statements are equivalent:

(1). $A_{n}\{1,3\} A_{n-1}\{1,3\} \cdots A_{1}\{1,3\} \subseteq\left(A_{1} A_{2} \cdots A_{n}\right)\{1\}$;

(2). $r\left(A_{1} A_{2} \cdots A_{n-1} F_{A_{n}^{*}}, A_{1} A_{2} \cdots A_{n-2} F_{A_{n-1}^{*}}, \ldots, A_{1} F_{A_{2}^{*}}\right)$ $=r\left(A_{1}\right)-r\left(A_{1} \cdots A_{n}\right)$;

(3). $r\left(\begin{array}{cccc}A_{n}^{*} & O & \cdots & O \\ O & A_{n-1}^{*} & \cdots & O \\ \vdots & \vdots & \ddots & \vdots \\ O & O & \cdots & A_{2}^{*} \\ A_{1} A_{2} \cdots A_{n-1} & A_{1} A_{2} \cdots A_{n-2} & \cdots & A_{1}\end{array}\right)=\sum_{i=1}^{n} r\left(A_{i}\right)-r\left(A_{1} A_{2} \cdots A_{n}\right)$.

EXAMPLE 3.3. Take

$$
A_{1}=\left(\begin{array}{ll}
1 & 0 \\
0 & 0
\end{array}\right), A_{2}=\left(\begin{array}{lll}
1 & 0 & 0 \\
1 & 1 & 0
\end{array}\right) \text { and } A_{3}=\left(\begin{array}{lll}
1 & 0 & 0 \\
0 & 1 & 1 \\
0 & 1 & 1
\end{array}\right) .
$$

We easily get

$$
r\left(\begin{array}{cc}
A_{3}^{*} & O \\
O & A_{2}^{*} \\
A_{1} A_{2} & A_{1}
\end{array}\right)+r\left(A_{1} A_{2} A_{3}\right)=r\left(A_{1}\right)+r\left(A_{2}\right)+r\left(A_{3}\right)=5 .
$$

This implies that the matrices $A_{1}, A_{2}$ and $A_{3}$ satisfy the condition (3) in Theorem 3.2. On the other hand, since (also by formula (2.13))

$$
\begin{aligned}
& A_{1}\{1,3\}=\left\{\left(\begin{array}{cc}
1 & 0 \\
a_{1} & b_{1}
\end{array}\right) \mid a_{1}, b_{1} \in \mathbb{C}\right\}, \\
& A_{2}\{1,3\}=\left\{\left(\begin{array}{cc}
1 & 0 \\
-1 & 1 \\
a_{2} & b_{2}
\end{array}\right) \mid a_{2}, b_{2} \in \mathbb{C}\right\}
\end{aligned}
$$


and

$$
A_{3}\{1,3\}=\left\{\left(\begin{array}{ccc}
1 & 0 & 0 \\
a_{3} & b_{3} & c \\
-a_{3} & 1 / 2-b_{3} & 1 / 2-c
\end{array}\right) \mid a_{3}, b_{3}, c \in \mathbb{C}\right\},
$$

we have

$$
\begin{aligned}
& A_{3}\{1,3\} A_{2}\{1,3\} A_{1}\{1,3\}=\left\{M_{2}:\right. \\
& \left.M_{2}=\left(\begin{array}{ccc}
1 & 0 & 0 \\
a_{3} & b_{3} & c \\
-a_{3} & 1 / 2-b_{3} & 1 / 2-c
\end{array}\right)\left(\begin{array}{cc}
1 & 0 \\
-1 & 1 \\
a_{2} & b_{2}
\end{array}\right)\left(\begin{array}{cc}
1 & 0 \\
a_{1} & b_{1}
\end{array}\right) \mid a_{i}, b_{j}, c \in \mathbb{C}\right\} .
\end{aligned}
$$

Similar to Example 2.3, we can easily check that the identity

$$
\left(A_{1} A_{2} A_{3}\right) M_{2}\left(A_{1} A_{2} A_{3}\right)=A_{1} A_{2} A_{3}
$$

holds for any matrix $M_{2} \in A_{3}\{1,3\} A_{2}\{1,3\} A_{1}\{1,3\}$. Hence,

$$
A_{3}\{1,3\} A_{2}\{1,3\} A_{1}\{1,3\} \subseteq\left(A_{1} A_{2} A_{3}\right)\{1\} .
$$

Again by the assertion that $G \in A\{1,4\}$ if and only if $G^{*} \in A^{*}\{1,3\}$, we can get the necessary and sufficient conditions for the inclusion (1.5) from the results obtained in Theorem 3.2.

Theorem 3.4. Let $A_{i} \in \mathbb{C}^{l_{i} \times l_{i+1}}, i=1,2, \ldots, n$. Then the following statements are equivalent:

(1). $A_{n}\{1,4\} A_{n-1}\{1,4\} \cdots A_{1}\{1,4\} \subseteq\left(A_{1} A_{2} \cdots A_{n}\right)\{1\}$;

$(2) \cdot r\left(\begin{array}{c}E_{A_{1}^{*} A_{2} A_{3} \cdots A_{n}} \\ E_{A_{2}^{*} A_{3} A_{4} \cdots A_{n}} \\ \vdots \\ E_{A_{n-1}^{*}} A_{n}\end{array}\right)+r\left(A_{1} A_{2} \cdots A_{n}\right)=r\left(A_{n}\right)$

(3). $r\left(\begin{array}{ccccc}A_{1}^{*} & O & \cdots & O & A_{2} A_{3} \cdots A_{n} \\ O & A_{2}^{*} & \cdots & O & A_{3} A_{4} \cdots A_{n} \\ \vdots & \vdots & \ddots & \vdots & \vdots \\ O & O & \cdots & A_{n-1}^{*} & A_{n}\end{array}\right)+r\left(A_{1} A_{2} \cdots A_{n}\right)=\sum_{i=1}^{n} r\left(A_{i}\right)$

4. The necessary and sufficient conditions for the inclusions (1.6) and (1.7). In order to present the necessary and sufficient condition for the inclusion (1.6), 
we first give the maximum rank of matrix function $T_{A_{1}, A_{2}, \ldots, A_{n}}\left(X_{1}, X_{2}, \ldots, X_{n}\right)$ when each $X_{i}(i=1,2, \ldots, n)$ varies over the set $A_{i}\{1\}$ of all $g$-inverses of the matrix $A_{i}$.

TheOREM 4.1. Let $A_{i} \in \mathbb{C}^{l_{i} \times l_{i+1}}, i=1, \ldots, n$ and $T_{A_{1}, A_{2}, \ldots, A_{n}}\left(X_{1}, X_{2}, \ldots, X_{n}\right)$ be as in (1.9). Then

$$
\begin{aligned}
& \max _{X_{n}, X_{n-1}, \ldots, X_{1}} r\left(T_{A_{1}, A_{2}, \ldots, A_{n}}\left(X_{1}, X_{2}, \ldots, X_{n}\right)\right) \\
= & \min \left\{r\left(A_{1} A_{2} \cdots A_{n}\right), \sum_{m=1}^{n} l_{m}-\sum_{m=1}^{n} r\left(A_{m}\right)\right\},
\end{aligned}
$$

where $X_{i}$ varies over $A_{i}\{1\}$ for $i=1,2, \ldots, n$.

Proof. Let $\mathscr{A}_{i}^{j}$ and $\mathscr{X}_{i}^{j}, 1 \leq i \leq j \leq n$, be as in (1.8). Then for $2 \leq i \leq n-1$ and $X_{j} \in A_{j}\{1\}, j=i, i+1, \ldots, n$, we first prove

$$
\begin{aligned}
& \max _{X_{i}} r\left(\left(\mathscr{A}_{1}^{n}\right)^{*} \mathscr{A}_{1}^{i-1}-\left(\mathscr{A}_{1}^{n}\right)^{*} \mathscr{A}_{1}^{n}\left(\mathscr{X}_{i}^{n}\right)^{*}\right) \\
= & \min \left\{r\left(\left(\mathscr{A}_{1}^{n}\right)^{*} \mathscr{A}_{1}^{i-1}\right), \quad r\left(\left(\mathscr{A}_{1}^{n}\right)^{*} \mathscr{A}_{1}^{i}-\left(\mathscr{A}_{1}^{n}\right)^{*} \mathscr{A}_{1}^{n}\left(\mathscr{X}_{i+1}^{n}\right)^{*}\right)+l_{i}-r\left(A_{i}\right)\right\} .
\end{aligned}
$$

By the formula (1.11) in Lemma 1.1 (with $A=A_{i}, B=I_{l_{i}}, C=\left(\mathscr{A}_{1}^{n}\right)^{*} \mathscr{A}_{1}^{n}\left(\mathscr{X}_{i+1}^{n}\right)^{*}$, $\left.D=\left(\mathscr{A}_{1}^{n}\right)^{*} \mathscr{A}_{1}^{i-1}\right)$, we have

$$
\begin{aligned}
& \max _{X_{i}} r\left(\left(\mathscr{A}_{1}^{n}\right)^{*} \mathscr{A}_{1}^{i-1}-\left(\mathscr{A}_{1}^{n}\right)^{*} \mathscr{A}_{1}^{n}\left(\mathscr{X}_{i}^{n}\right)^{*}\right) \\
& =\min \left\{r\left(\left(\mathscr{A}_{1}^{n}\right)^{*} \mathscr{A}_{1}^{n}\left(\mathscr{X}_{i+1}^{n}\right)^{*}, \quad\left(\mathscr{A}_{1}^{n}\right)^{*} \mathscr{A}_{1}^{i-1}\right), \quad r\left(\begin{array}{c}
I_{l_{i}} \\
\left(\mathscr{A}_{1}^{n}\right)^{*} \mathscr{A}_{1}^{i-1}
\end{array}\right),\right. \\
& \left.r\left(\begin{array}{cc}
A_{i} & I_{l_{i}} \\
\left(\mathscr{A}_{1}^{n}\right)^{*} \mathscr{A}_{1}^{n}\left(\mathscr{X}_{i+1}^{n}\right)^{*} & \left(\mathscr{A}_{1}^{n}\right)^{*} \mathscr{A}_{1}^{i-1}
\end{array}\right)-r\left(A_{i}\right)\right\} \\
& =\min \left\{r\left(\left(\mathscr{A}_{1}^{n}\right)^{*} \mathscr{A}_{1}^{n}\left(\mathscr{X}_{i+1}^{n}\right)^{*}, \quad\left(\mathscr{A}_{1}^{n}\right)^{*} \mathscr{A}_{1}^{i-1}\right), \quad r\left(\begin{array}{c}
I_{l_{i}} \\
\left(\mathscr{A}_{1}^{n}\right)^{*} \mathscr{A}_{1}^{i-1}
\end{array}\right),\right. \\
& \left.r\left(\left(\mathscr{A}_{1}^{n}\right)^{*} \mathscr{A}_{1}^{i}-\left(\mathscr{A}_{1}^{n}\right)^{*} \mathscr{A}_{1}^{n}\left(\mathscr{X}_{i+1}^{n}\right)^{*}\right)+l_{i}-r\left(A_{i}\right)\right\} \\
& =\min \left\{r\left(\left(\mathscr{A}_{1}^{n}\right)^{*} \mathscr{A}_{1}^{i-1}\right), \quad r\left(\left(\mathscr{A}_{1}^{n}\right)^{*} \mathscr{A}_{1}^{i}-\left(\mathscr{A}_{1}^{n}\right)^{*} \mathscr{A}_{1}^{n}\left(\mathscr{X}_{i+1}^{n}\right)^{*}\right)+l_{i}-r\left(A_{i}\right)\right\} \text {, }
\end{aligned}
$$

i.e., (4.2) holds, where the second equality holds as

$$
\begin{aligned}
& r\left(\begin{array}{cc}
A_{i} & I_{l_{i}} \\
\left(\mathscr{A}_{1}^{n}\right)^{*} \mathscr{A}_{1}^{n}\left(\mathscr{X}_{i+1}^{n}\right)^{*} & \left(\mathscr{A}_{1}^{n}\right)^{*} \mathscr{A}_{1}^{i-1}
\end{array}\right) \\
& \quad=r\left(\begin{array}{cc}
O & I_{l_{i}} \\
\left(\mathscr{A}_{1}^{n}\right)^{*} \mathscr{A}_{1}^{n}\left(\mathscr{X}_{i+1}^{n}\right)^{*}-\left(\mathscr{A}_{1}^{n}\right)^{*} \mathscr{A}_{1}^{i} & O
\end{array}\right) .
\end{aligned}
$$

The last equality holds as

$$
r\left(\left(\mathscr{A}_{1}^{n}\right)^{*} \mathscr{A}_{1}^{n}\left(\mathscr{X}_{i+1}^{n}\right)^{*}, \quad\left(\mathscr{A}_{1}^{n}\right)^{*} \mathscr{A}_{1}^{i-1}\right)=r\left(\left(\mathscr{A}_{1}^{n}\right)^{*} \mathscr{A}_{1}^{i-1}\right)
$$


and

$$
r\left(\left(\mathscr{A}_{1}^{n}\right)^{*} \mathscr{A}_{1}^{i-1}\right) \leq r\left(A_{i-1}\right) \leq l_{i}=r\left(\begin{array}{c}
I_{l_{i}} \\
\left(\mathscr{A}_{1}^{n}\right)^{*} \mathscr{A}_{1}^{i-1}
\end{array}\right) .
$$

When $i=n$, again by Lemma 1.1 (1.11) with $A=A_{n}, B=I_{l_{n}}, D=\left(\mathscr{A}_{1}^{n}\right)^{*} \mathscr{A}_{1}^{n-1}$ and $C=\left(\mathscr{A}_{1}^{n}\right)^{*} \mathscr{A}_{1}^{n}$, we have

$$
\begin{aligned}
& \max _{X_{n}} r\left(\left(\mathscr{A}_{1}^{n}\right)^{*} \mathscr{A}_{1}^{n-1}-\left(\mathscr{A}_{1}^{n}\right)^{*} \mathscr{A}_{1}^{n}\left(\mathscr{X}_{n}^{n}\right)^{*}\right) \\
= & \min \left\{r\left(\left(\mathscr{A}_{1}^{n}\right)^{*} \mathscr{A}_{1}^{n}, \quad\left(\mathscr{A}_{1}^{n}\right)^{*} \mathscr{A}_{1}^{n-1}\right), r\left(\begin{array}{c}
I_{l_{n}} \\
\left(\mathscr{A}_{1}^{n}\right)^{*} \mathscr{A}_{1}^{n-1}
\end{array}\right),\right. \\
& \left.r\left(\begin{array}{cc}
A_{n} & I_{l_{n}} \\
\left(\mathscr{A}_{1}^{n}\right)^{*} \mathscr{A}_{1}^{n} & \left(\mathscr{A}_{1}^{n}\right)^{*} \mathscr{A}_{1}^{n-1}
\end{array}\right)-r\left(A_{n}\right)\right\} \\
= & \min \left\{r\left(\left(\mathscr{A}_{1}^{n}\right)^{*} \mathscr{A}_{1}^{n-1}\right), \quad l_{n}-r\left(A_{n}\right)\right\}
\end{aligned}
$$

in which the last equality holds since

$$
\begin{gathered}
r\left(\left(\mathscr{A}_{1}^{n}\right)^{*} \mathscr{A}_{1}^{n}, \quad\left(\mathscr{A}_{1}^{n}\right)^{*} \mathscr{A}_{1}^{n-1}\right)=r\left(\left(\mathscr{A}_{1}^{n}\right)^{*} \mathscr{A}_{1}^{n-1}\right), \\
r\left(\left(\mathscr{A}_{1}^{n}\right)^{*} \mathscr{A}_{1}^{n-1}\right) \leq r\left(A_{n-1}\right) \leq l_{n}=r\left(\begin{array}{c}
I_{l_{n}} \\
\left(\mathscr{A}_{1}^{n}\right)^{*} A \mathscr{A}_{1}^{n-1}
\end{array}\right)
\end{gathered}
$$

and

$$
r\left(\begin{array}{cc}
A_{n} & I_{l_{n}} \\
\left(\mathscr{A}_{1}^{n}\right)^{*} \mathscr{A}_{1}^{n} & \left(\mathscr{A}_{1}^{n}\right)^{*} \mathscr{A}_{1}^{n-1}
\end{array}\right)=r\left(\begin{array}{c}
I_{l_{n}} \\
\left(\mathscr{A}_{1}^{n}\right)^{*} \mathscr{A}_{1}^{n-1}
\end{array}\right) .
$$

We now prove (4.1). According to Lemma 1.1 (1.11) with $A=A_{1}, B=I_{l_{1}}$, $C=\left(\mathscr{A}_{1}^{n}\right)^{*} \mathscr{A}_{1}^{n}\left(\mathscr{X}_{2}^{n}\right)^{*}$ and $D=\left(\mathscr{A}_{1}^{n}\right)^{*}$, we have

$$
\begin{aligned}
& \max _{X_{1}} r\left(T_{A_{1}, A_{2}, \ldots, A_{n}}\left(X_{1}, X_{2}, \ldots, X_{n}\right)\right) \\
= & \min \left\{r\left(\left(\mathscr{A}_{1}^{n}\right)^{*} \mathscr{A}_{1}^{n}\left(\mathscr{X}_{2}^{n}\right)^{*}, \quad\left(\mathscr{A}_{1}^{n}\right)^{*}\right), r\left(\begin{array}{c}
I_{l_{1}} \\
\left(\mathscr{A}_{1}^{n}\right)^{*}
\end{array}\right),\right. \\
& \left.r\left(\begin{array}{cc}
A_{1} & I_{l_{1}} \\
\left(\mathscr{A}_{1}^{n}\right)^{*} \mathscr{A}_{1}^{n}\left(\mathscr{X}_{2}^{n}\right)^{*} & \left(\mathscr{A}_{1}^{n}\right)^{*}
\end{array}\right)-r\left(A_{1}\right)\right\} \\
= & \min \left\{r\left(\mathscr{A}_{1}^{n}\right), \quad r\left(\left(\mathscr{A}_{1}^{n}\right)^{*} \mathscr{A}_{1}^{1}-\left(\mathscr{A}_{1}^{n}\right)^{*} \mathscr{A}_{1}^{n}\left(\mathscr{X}_{2}^{n}\right)^{*}\right)+l_{1}-r\left(A_{1}\right)\right\},
\end{aligned}
$$

where the last equality holds as

$$
r\left(\left(\mathscr{A}_{1}^{n}\right)^{*} \mathscr{A}_{1}^{n}\left(\mathscr{X}_{2}^{n}\right)^{*}, \quad\left(\mathscr{A}_{1}^{n}\right)^{*}\right)=r\left(\mathscr{A}_{1}^{n}\right) \leq r\left(\begin{array}{c}
I_{l_{1}} \\
\left(\mathscr{A}_{1}^{n}\right)^{*}
\end{array}\right)
$$


and

$$
r\left(\begin{array}{cc}
A_{1} & I_{l_{1}} \\
\left(\mathscr{A}_{1}^{n}\right)^{*} \mathscr{A}_{1}^{n}\left(\mathscr{X}_{2}^{n}\right)^{*} & \left(\mathscr{A}_{1}^{n}\right)^{*}
\end{array}\right)=r\left(\left(\mathscr{A}_{1}^{n}\right)^{*} \mathscr{A}_{1}^{1}-\left(\mathscr{A}_{1}^{n}\right)^{*} \mathscr{A}_{1}^{n}\left(\mathscr{X}_{2}^{n}\right)^{*}\right)+l_{1} .
$$

Combining (4.2) and (4.4), we have

$$
\begin{aligned}
& \max _{X_{2}, X_{1}} r\left(T_{A_{1}, A_{2}, \ldots, A_{n}}\left(X_{1}, X_{2}, \ldots, X_{n}\right)\right) \\
= & \min \left\{r\left(\mathscr{A}_{1}^{n}\right), \quad \max _{X_{2}} r\left(\left(\mathscr{A}_{1}^{n}\right)^{*} \mathscr{A}_{1}^{1}-\left(\mathscr{A}_{1}^{n}\right)^{*} \mathscr{A}_{1}^{n}\left(\mathscr{X}_{2}^{n}\right)^{*}\right)+l_{1}-r\left(A_{1}\right)\right\} \\
= & \min \left\{r\left(\mathscr{A}_{1}^{n}\right), \quad r\left(\left(\mathscr{A}_{1}^{n}\right)^{*} \mathscr{A}_{1}^{1}\right)+l_{1}-r\left(A_{1}\right), r\left(\left(\mathscr{A}_{1}^{n}\right)^{*} \mathscr{A}_{1}^{2}-\left(\mathscr{A}_{1}^{n}\right)^{*} \mathscr{A}_{1}^{n}\left(\mathscr{X}_{3}^{n}\right)^{*}\right)\right. \\
& \left.+l_{2}+l_{1}-r\left(A_{2}\right)-r\left(A_{1}\right)\right\} \\
= & \min \left\{r\left(\mathscr{A}_{1}^{n}\right), \quad r\left(\left(\mathscr{A}_{1}^{n}\right)^{*} \mathscr{A}_{1}^{2}-\left(\mathscr{A}_{1}^{n}\right)^{*} \mathscr{A}_{1}^{n}\left(\mathscr{X}_{3}^{n}\right)^{*}\right)+l_{2}+l_{1}-r\left(A_{2}\right)-r\left(A_{1}\right)\right\},
\end{aligned}
$$

in which the last equality holds since from Lemma 1.3, we have

$$
r\left(\left(\mathscr{A}_{1}^{n}\right)^{*} \mathscr{A}_{1}^{1}\right)+l_{1}-r\left(A_{1}\right) \geq r\left(\left(\mathscr{A}_{1}^{n}\right)^{*}\right)+r\left(A_{1}\right)-r\left(A_{1}\right)=r\left(\mathscr{A}_{1}^{n}\right) .
$$

We contend that, for $2 \leq i \leq n-1$,

$$
\text { 5) } \begin{aligned}
& \max _{X_{i}, X_{i-1}, \ldots, X_{1}} r\left(S_{A_{1}, A_{2}, \ldots, A_{n}}\left(X_{1}, X_{2}, \ldots, X_{n}\right)\right) \\
= & \min \left\{r\left(\mathscr{A}_{1}^{n}\right), r\left(\left(\mathscr{A}_{1}^{n}\right)^{*} \mathscr{A}_{1}^{i}-\left(\mathscr{A}_{1}^{n}\right)^{*} \mathscr{A}_{1}^{n}\left(\mathscr{X}_{i+1}^{n}\right)^{*}\right)+\sum_{m=1}^{i} l_{m}-\sum_{m=1}^{i} r\left(A_{m}\right)\right\} .
\end{aligned}
$$

We proceed by induction on $i$. For $i=2$, the equality relation (4.5) has been proved. Assume that (4.5) is true for $i-1(i \geq 3)$, that is

$$
\text { 6) } \begin{aligned}
& \max _{X_{i-1}, X_{i-2}, \ldots, X_{1}} r\left(T_{A_{1}, A_{2}, \ldots, A_{n}}\left(X_{1}, X_{2}, \ldots, X_{n}\right)\right) \\
= & \min \left\{r\left(\mathscr{A}_{1}^{n}\right), r\left(\left(\mathscr{A}_{1}^{n}\right)^{*} \mathscr{A}_{1}^{i-1}-\left(\mathscr{A}_{1}^{n}\right)^{*} \mathscr{A}_{1}^{n}\left(\mathscr{X}_{i}^{n}\right)^{*}\right)+\sum_{m=1}^{i-1} l_{m}-\sum_{m=1}^{i-1} r\left(A_{m}\right)\right\} .
\end{aligned}
$$

We now prove that (4.5) is also true for $i$. By (4.2) and (4.6), we have

$$
\begin{aligned}
& \max _{X_{i}, X_{i-1}, \ldots, X_{1}} r\left(T_{A_{1}, A_{2}, \ldots, A_{n}}\left(X_{1}, X_{2}, \ldots, X_{n}\right)\right) \\
= & \min \left\{r\left(\mathscr{A}_{1}^{n}\right), \quad \max _{X_{i}} r\left(\left(\mathscr{A}_{1}^{n}\right)^{*} \mathscr{A}_{1}^{i-1}-\left(\mathscr{A}_{1}^{n}\right)^{*} \mathscr{A}_{1}^{n}\left(\mathscr{X}_{i}^{n}\right)^{*}\right)+\sum_{m=1}^{i-1} l_{m}-\sum_{m=1}^{i-1} r\left(A_{m}\right)\right\} \\
= & \min \left\{r\left(\mathscr{A}_{1}^{n}\right), \quad r\left(\left(\mathscr{A}_{1}^{n}\right)^{*} \mathscr{A}_{1}^{i-1}\right)+\sum_{m=1}^{i-1} l_{m}-\sum_{m=1}^{i-1} r\left(A_{m}\right),\right. \\
& \left.r\left(\left(\mathscr{A}_{1}^{n}\right)^{*} \mathscr{A}_{1}^{i}-\left(\mathscr{A}_{1}^{n}\right)^{*} \mathscr{A}_{1}^{n}\left(\mathscr{X}_{i+1}^{n}\right)^{*}\right)+l_{i}-r\left(A_{i}\right)+\sum_{m=1}^{i-1} l_{m}-\sum_{m=1}^{i-1} r\left(A_{m}\right)\right\} .
\end{aligned}
$$


From Lemma 1.3, we know

$$
r\left(\left(\mathscr{A}_{1}^{n}\right)^{*} \mathscr{A}_{1}^{i-1}\right)+\sum_{m=1}^{i-1} l_{m} \geq r\left(\mathscr{A}_{1}^{n}\right)+\sum_{m=1}^{i-1} r\left(A_{m}\right),
$$

thus

$$
\begin{aligned}
& \max _{X_{i}, X_{i-1}, \ldots, X_{1}} r\left(T_{A_{1}, A_{2}, \ldots, A_{n}}\left(X_{1}, X_{2}, \ldots, X_{n}\right)\right) \\
= & \min \left\{r\left(\mathscr{A}_{1}^{n}\right), \quad r\left(\left(\mathscr{A}_{1}^{n}\right)^{*} \mathscr{A}_{1}^{i}-\left(\mathscr{A}_{1}^{n}\right)^{*} \mathscr{A}_{1}^{n}\left(\mathscr{X}_{i+1}^{n}\right)^{*}\right)+\sum_{m=1}^{i} l_{m}-\sum_{m=1}^{i} r\left(A_{m}\right)\right\} .
\end{aligned}
$$

In particular, when $i=n-1$, we have

$$
\begin{aligned}
& \max _{X_{n-1}, X_{n-2}, \ldots, X_{1}} r\left(T_{A_{1}, A_{2}, \ldots, A_{n}}\left(X_{1}, X_{2}, \ldots, X_{n}\right)\right) \\
= & \min \left\{r\left(\mathscr{A}_{1}^{n}\right), \quad r\left(\left(\mathscr{A}_{1}^{n}\right)^{*} \mathscr{A}_{1}^{n-1}-\left(\mathscr{A}_{1}^{n}\right)^{*} \mathscr{A}_{1}^{n}\left(\mathscr{X}_{n}^{n}\right)^{*}\right)+\sum_{m=1}^{n-1} l_{m}-\sum_{m=1}^{n-1} r\left(A_{m}\right)\right\} .
\end{aligned}
$$

On account of (4.3) and (4.7), it is seen that

$$
\begin{aligned}
& \max _{X_{n}, X_{n-1}, \ldots, X_{1}} r\left(T_{A_{1}, A_{2}, \ldots, A_{n}}\left(X_{1}, X_{2}, \ldots, X_{n}\right)\right) \\
= & \min \left\{r\left(\mathscr{A}_{1}^{n}\right), \quad \max _{X_{n}} r\left(\left(\mathscr{A}_{1}^{n}\right)^{*} \mathscr{A}_{1}^{n-1}-\left(\mathscr{A}_{1}^{n}\right)^{*} \mathscr{A}_{1}^{n}\left(\mathscr{X}_{n}^{n}\right)^{*}\right)+\sum_{m=1}^{n-1} l_{m}-\sum_{m=1}^{n-1} r\left(A_{m}\right)\right\} \\
= & \min \left\{r\left(\mathscr{A}_{1}^{n}\right), \quad r\left(\left(\mathscr{A}_{1}^{n}\right)^{*} \mathscr{A}_{1}^{n-1}\right)+\sum_{m=1}^{n-1} l_{m}-\sum_{m=1}^{n-1} r\left(A_{m}\right),\right. \\
& \left.l_{n}-r\left(A_{n}\right)+\sum_{m=1}^{n-1} l_{m}-\sum_{m=1}^{n-1} r\left(A_{m}\right)\right\} .
\end{aligned}
$$

Noting that

$$
r\left(\left(\mathscr{A}_{1}^{n}\right)^{*} \mathscr{A}_{1}^{n-1}\right)+\sum_{m=1}^{n-1} l_{m} \geq r\left(\mathscr{A}_{1}^{n}\right)+\sum_{m=1}^{n-1} r\left(A_{m}\right),
$$

we finally have

$$
\begin{aligned}
& \max _{X_{n}, X_{n-1}, \ldots, X_{1}} r\left(T_{A_{1}, A_{2}, \ldots, A_{n}}\left(X_{1}, X_{2}, \ldots, X_{n}\right)\right) \\
= & \min \left\{r\left(A_{1} A_{2} \cdots A_{n}\right), \sum_{m=1}^{n} l_{m}-\sum_{m=1}^{n} r\left(A_{m}\right)\right\} .
\end{aligned}
$$

Since the inclusion (1.6) holds if and only if

$$
\max _{X_{n}, X_{n-1}, \ldots, X_{1}} r\left(T_{A_{1}, A_{2}, \ldots, A_{n}}\left(X_{1}, X_{2}, \ldots, X_{n}\right)\right)=0,
$$


by Theorem 4.1 and Lemma 1.2 (1.14), we can immediately obtain the following result:

Theorem 4.2. Let $A_{i} \in \mathbb{C}^{l_{i} \times l_{i+1}}, i=1,2, \ldots, n$. Then the inclusion (1.6) holds if and only if

$$
\min \left\{r\left(A_{1} A_{2} \cdots A_{n}\right), \sum_{m=1}^{n} l_{m}-\sum_{m=1}^{n} r\left(A_{m}\right)\right\}=0
$$

that is,

$$
A_{1} A_{2} \cdots A_{n}=O \quad \text { or } \quad \sum_{m=1}^{n} l_{m}=\sum_{m=1}^{n} r\left(A_{m}\right), \text { i.e., } \quad r\left(A_{i}\right)=l_{i}, \quad i=1,2, \ldots, n \text {. }
$$

EXAMPLE 4.3. Let

$$
A_{1}=(1,0), A_{2}=\left(\begin{array}{lll}
1 & 1 & 1 \\
0 & 1 & 0
\end{array}\right) \text { and } A_{3}=\left(\begin{array}{llll}
1 & 0 & 0 & 1 \\
0 & 1 & 0 & 0 \\
0 & 0 & 1 & 0
\end{array}\right)
$$

Then $r\left(A_{1}\right)=1, r\left(A_{2}\right)=2$ and $r\left(A_{3}\right)=3$, and the ranks of these matrices satisfy the conditions in Theorem 4.2 .

On the other hand, by the definition of $\{1\}$-inverse, we have

$$
\begin{gathered}
A_{3}\{1\}=\left\{\left(\begin{array}{ccc}
a_{1} & a_{2} & a_{3} \\
0 & 1 & 0 \\
0 & 0 & 1 \\
1-a_{1} & -a_{2} & -a_{3}
\end{array}\right) \mid a_{1}, a_{2}, a_{3} \in \mathbb{C}\right\}, \\
A_{2}\{1\}=\left\{\left(\begin{array}{cc}
b_{1} & b_{2} \\
0 & 1 \\
1-b_{1} & -1-b_{2}
\end{array}\right) \mid b_{1}, b_{2} \in \mathbb{C}\right\} \text { and } A_{1}\{1\}=\left\{\left(\begin{array}{c}
1 \\
c_{1}
\end{array}\right) \mid c_{1} \in \mathbb{C}\right\} .
\end{gathered}
$$

Hence

$$
\begin{aligned}
& A_{3}\{1\} A_{2}\{1\} A\{1\}=\left\{M_{3}:\right. \\
& \left.M_{3}=\left(\begin{array}{ccc}
a_{1} & a_{2} & a_{3} \\
0 & 1 & 0 \\
0 & 0 & 1 \\
1-a_{1} & -a_{2} & -a_{3}
\end{array}\right)\left(\begin{array}{cc}
b_{1} & b_{2} \\
0 & 1 \\
1-b_{1} & -1-b_{2}
\end{array}\right)\left(\begin{array}{c}
1 \\
c_{1}
\end{array}\right) \mid a_{i}, b_{j}, c_{k} \in \mathbb{C}\right\} .
\end{aligned}
$$

It is easy to verify that if $M_{3} \in A_{3}\{1\} A_{2}\{1\} A\{1\}$, then

$$
\left(A_{1} A_{2} A_{3}\right) M_{3}\left(A_{1} A_{2} A_{3}\right)=A_{1} A_{2} A_{3} \text { and }\left(A_{1} A_{2} A_{3} M_{3}\right)^{*}=A_{1} A_{2} A_{3} M_{3} \text {. }
$$


Hence

$$
A_{3}\{1\} A_{2}\{1\} A_{1}\{1\} \subseteq\left(A_{1} A_{2} A_{3}\right)\{1,3\}
$$

By Lemma $1.2(1.14)$ and (1.15), $G \in A\{1,4\}$ if and only if $G^{*} \in A^{*}\{1,3\}$. So from the results obtained in Theorem 4.2 , we can get the necessary and sufficient conditions for the inclusion (1.7).

Theorem 4.4. Let $A_{i} \in \mathbb{C}^{l_{i} \times l_{i+1}}, i=1,2, \ldots, n$. Then the inclusion (1.7) holds if and only if

$$
\min \left\{r\left(A_{1} A_{2} \cdots A_{n}\right), \sum_{m=2}^{n+1} l_{m}-\sum_{m=1}^{n} r\left(A_{m}\right)\right\}=0
$$

that is,

$$
A_{1} A_{2} \cdots A_{n}=O \quad \text { or } \quad \sum_{m=2}^{n+1} l_{m}=\sum_{m=1}^{n} r\left(A_{m}\right), \text { i.e., } r\left(A_{i}\right)=l_{i+1}, \quad i=1,2, \ldots, n \text {. }
$$

Acknowledgment. The authors are very grateful to Prof. O.M. Baksalary and the anonymous referees for their valuable comments and suggestions, which lead to an improvement of the original manuscript.

\section{REFERENCES}

[1] D.T. Barwick and J.D. Gilbert. On generalizations of the reverse order law. SIAM J. Appl. Math., 27:326-330, 1974.

[2] A. Ben-Israel and T.N.E. Greville. Generalized Inverses: Theory and Applications. WileyInterscience, 1974; 2nd Edition, Springer-Verlag, New York, 2002.

[3] D.S. Djordjević. Further results on the reverse order law for generalized inverses. SIAM J. Matrix Anal. Appl., 29(4):1242-1246, 2007.

[4] T.N.E. Greville. Note on the generalized inverse of a matrix product. SIAM Rev., 8:518-521, 1966.

[5] R.E. Hartwig. The reverse order law revisited. Linear Algebra Appl., 76:241-246, 1986.

[6] Q. Liu and M. Wei. Reverse order law for least squares $g$-inverses of multiple matrix products. Linear Multilinear Algebra, 56(5):491-506, 2008.

[7] G. Marsaglia and G.P.H. Styan. Equalities and inequalities for ranks of matrices. Linear Multilinear Algebra, 2:269-292, 1974.

[8] W. Sun and Y. Wei. Inverse order rule for weighted generalized inverse. SIAM J. Matrix Anal. Appl., 19:772-775, 1998.

[9] W. Sun and Y. Wei. Triple reverse-order law for weighted generalized inverses. Appl. Math. Comput., 125:221-229, 2002.

[10] Y. Tian. Reverse order laws for the generalized inverses of multiple matrix products. Linear Algebra Appl., 211:85-100, 1994. 
[11] Y. Tian. Upper and lower bounds for ranks of matrix expressions using generalized inverses. Linear Algebra Appl., 355:187-214, 2002.

[12] Y. Tian. More on maximal and minimal ranks of Schur complements with applications. Appl. Math. Comput., 152:675-692, 2004.

[13] Y. Tian. On mixed-type reverse order laws for the Moore-Penrose inverse of a matrix product. Int. J. Math. Math. Sci., 58:3103-3116, 2004.

[14] Y. Tian and Y. Liu. On a group of mixed-type reverse order laws for generalized inverses of a triple matrix product with applications. Electron. J. Linear Algebra, 16:73-89, 2007.

[15] G. Wang, Y. Wei, and S. Qiao. Generalized Inverses: Theory and Computations. Science Press, Beijing, 2004

[16] G. Wang and B. Zheng. The reverse order law for the generalized inverse $A_{T, S}^{(2)}$. Appl. Math. Comput., 157:295-305, 2004.

[17] M. Wei. Reverse order laws for generalized inverse of multiple matrix products. Linear Algebra Appl., 293:273-288, 1999.

[18] Z. Xiong and C. Jiang. The mixed-type reverse order laws for weighted generalized inverses of a triple matrix product. J. Appl. Math. Comput., 29:53-66, 2009.

[19] Z. Xiong and B. Zheng. The reverse order laws for $\{1,2,3\}$ - and $\{1,2,4\}$-inverses of two matrix product. Appl. Math. Lett., 21:649-655, 2008.

[20] B. Zheng and Z. Xiong. A new equivalent condition of the reverse order law for $G$-inverses of multiple matrix products. Electron. J. Linear Algebra, 17:1-8, 2008.

[21] B. Zheng and Z. Xiong. The reverse order laws for $\{1,2,3\}$ - and $\{1,2,4\}$-inverses of multiple matrix products. Linear Multilinear Algebra, 58(6):765-782, 2010. 